\title{
Ideal Gas Reference for Association and Dissociation Reactions: I. Basic Concepts
}

\author{
Tobias Binninger, ${ }^{1,}$ a) Adrian Heinritz, ${ }^{2}$ and Rhiyaad Mohamed $^{3}$
}

1) ICGM, Univ Montpellier, CNRS, ENSCM, Montpellier,

\section{France}

2) Electrochemistry Laboratory, Paul Scherrer Institut, 5232 Villigen, Switzerland

3) HySA/Catalysis Centre of Competence, Catalysis Institute, Department of Chemical Engineering, University of Cape Town, 7701, South Africa

Starting with a distance-based definition of molecules consisting of non-interacting atoms, which is in line with IUPAC terminology, we construct an ideal gas reference for chemical association and dissociation reactions. The corresponding ideal equations for equilibria and kinetics reveal the mathematical structure, known for real systems, in comprehensible clarity. The ideal gas reference corresponds to the limit of an entirely flat potential energy surface of the system where chemical equilibria and kinetics are determined by "unspecific" particle number combinatorics according to the reaction stoichiometry. The ideal equilibrium and rate constants provide a reference for the definition of excess equilibrium and rate constants of real reactions that quantify all "system-specific" contributions resulting from the particular shape of the potential energy surface. The ideal gas reference therefore enables a distinction between unspecific and system-specific aspects in the equilibria and kinetics of chemical association/dissociation reactions. Whereas conventional equilibrium and rate constants suffer from incompatibility between reactions of different stoichiometry, excess equilibrium and rate constants can be consistently compared across different reaction orders. Furthermore, whereas the conventional treatment requires an arbitrary specification of reference concentrations, e.g. at standard conditions, the ideal gas framework introduces an intrinsic concentration scale that is equal to the inverse of a molecular volume.

\footnotetext{
a)Electronic mail: tobias.binninger.science@gmx.de
} 


\section{INTRODUCTION}

At the very basis of chemistry lies the concept of a chemical reaction, which is the transformation of a number of chemical species, the reactants, into a number of other chemical species, the products. According to IUPAC terminology, a chemical species is an "ensemble of chemically identical molecular entities" ${ }^{1}$. Hence, the edifice of chemistry rests on the notion of a molecular entity, which IUPAC defines as a "constitutionally [...] distinct atom, molecule, [...] etc., identifiable as a separately distinguishable entity" ${ }^{1}$. This definition invokes a spatially distinct arrangement of the molecular entity's constituents, e.g. atoms, in a way that allows to identify the molecular entity as being separate from other entities. Clearly, this requires the constituents being "close" to each other and "far" from other entities. The idea of a molecular entity thus implicitly relies on a certain critical distance, or length scale, below which different constituents form a molecular entity, and above which they are considered to be separate. Such critical distance would naturally be of the order

of a chemical bond length so in the range of a few Å. In addition to the spatial aspect, the temporal dimension indirectly matters, because, in order to be identifiable, the question of the lifetime of a molecular entity arises. Throughout this work, we will use the terms "constituents" and "atoms" interchangeably, and the same for the terms "molecular entity" and "molecule". It is important to note that, in this context, the term "atom" is not limited to an atom in the strict sense, but it denotes any clearly defined constituent unit of a molecular entity. In particular, the term "atom" can also stand for an own molecular entity if the latter represents a well distinguishable building block of a larger molecular entity, e.g. the ligands of a complex.

Based on this reasoning, we define an ideal gas reference for chemical association and dissociation reactions. The ideal gas is the archetype model in thermodynamics, and thermodynamic quantities of real systems are commonly referenced to ideal gas behavior. However, no comparable archetype model exists for association and dissociation reactions in chemistry. In the present Part I. of this series, we propose an ideal gas model for chemical association/dissociation reactions. We first define molecular entities in ideal gas mixtures and derive an ideal law of mass action. Then, we turn to the ideal kinetics of association and dissociation to find a particularly symmetric structure of the rate equations for elementary reactions of different molecularity. Subsequently, we discuss a simple isotropic 
transition state model to include reaction barriers and binding energies into our consideration. This model precisely reproduces the equations for equilibrium and kinetics of the ideal gas reference in the limit of zero activation and binding energy. The ideal gas reference thus represents the limit of an entirely flat potential energy landscape. Turning to real association/dissociation reactions, we define excess equilibrium constants and excess rate constants that measure the deviation from ideal gas reference and quantify the effect of the unevenness of the system-specific potential energy surface. Remarkably, we find a renormalization scheme that makes rate constants comparable between different reaction orders. In this way, the ideal gas reference subtly cures an unsatisfactory aspect of the typical formulation of kinetic rate equations that produces incompatible rate constants for different reaction orders. Finally, we highlight the symmetry between the ideal gas reference for volume reactions and the typical treatment of surface reactions. In Part II. of our series, we will discuss the relevance of the ideal gas reference for electrochemical association/dissociation reactions, in particular the electrolysis of water.

\section{THEORY}

\section{A. Chemistry in Ideal Gases: Equilibrium}

To begin with, we pose a philosophical question: Does it make sense to define molecules $\mathrm{AB}$ consisting of non-interacting atoms $\mathrm{A}$ and $\mathrm{B}$ ? Intuitively, one would rather answer with "No". If atoms A and B do not interact, they cannot form chemical bonds, and thus no molecules. However, we begin with a gedankenexperiment and give the counterintuitive answer "Yes". Following the reasoning given in the Introduction, we simply define a molecule $\mathrm{AB}$ as any pair of atoms $\mathrm{A}$ and $\mathrm{B}$ that are closer than some critical distance $\ell_{\mathrm{ch}}$. We implicitly include in this definition that no additional atoms must be within the same distance, otherwise the molecule would not be denoted $\mathrm{AB}$ but rather $\mathrm{AB} X$ including all other $X$ constituents. Reasonable choices of $\ell_{\mathrm{ch}}$ will be of the order of a few $\AA$ as discussed further below. For now, we merely acknowledge that there exists a certain "chemical length scale" $\ell_{\text {ch }}$ at which matter is microscopically ordered, defining whether atomic constituents belong together or are to be considered as separate. This simple definition of molecules also works for the case of non-interacting atoms: Any time, along their individual trajectories, 


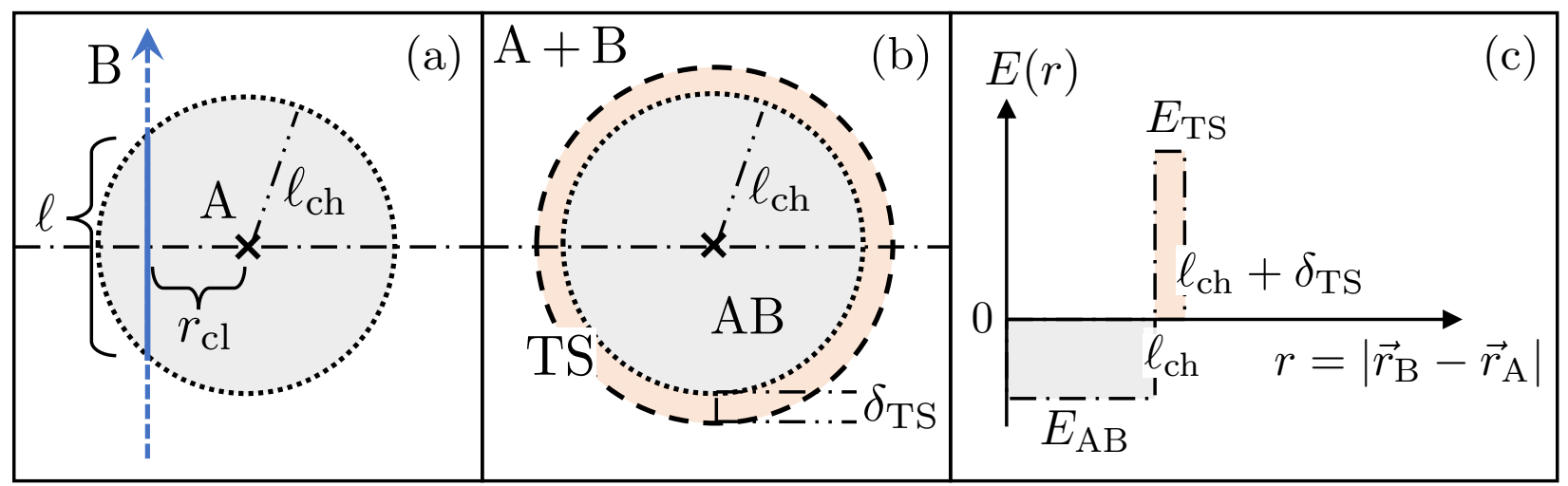

FIG. 1. (a) Trajectory of atom B in the rest frame of atom A, both of which taken as point-like ideal gas particles. For the time interval that their distance is less than $\ell_{\mathrm{ch}}$, the pair is defined to form a molecule AB. The gray-shaded circular area indicates the molecular region, $\ell$ is the length of the trajectory segment therein, and $r_{\mathrm{cl}}$ is the distance of closest approach. (b) Schematic of the isotropic transition-state model represented in the relative coordinate $\vec{r}=\vec{r}_{\mathrm{B}}-\vec{r}_{\mathrm{A}}$ of the atom pair. The transition state (TS) corresponds to a spherical shell around the spherical volume of the AB molecule. Outside the TS shell, both atoms are considered to be free (A+B). (c) Potential energy $E(r)$ as a function of the reaction coordinate $r=\left|\vec{r}_{\mathrm{B}}-\vec{r}_{\mathrm{A}}\right|$ for the isotropic TS model.

two atoms $\mathrm{A}$ and $\mathrm{B}$ get closer to each other than $\ell_{\mathrm{ch}}$, we call the pair a molecule $\mathrm{AB}$ as schematically visualized in Figure 1a. Obviously, the lifetime of such molecules will be extremely short, because the atoms simply "fly by" in the absence of interactions.

What is the equilibrium concentration of such molecules $\mathrm{AB}$ in a mixture of ideal gases A and B? For the sake of clarity, we exclusively focus on the constituent atom pair in the present example and the resulting equations (1) and (2) must be regarded as approximations. The influence of all other atoms of the gas mixture will be included in the general mathematical treatment in the subsequent paragraph. We assume point-like ideal gas particles that do not interact with each other. For a total of $N_{\mathrm{A}}^{\text {tot }}$ atoms of $\mathrm{A}$ and $N_{\mathrm{B}}^{\text {tot }}$ atoms of $\mathrm{B}$, there are a total of $N_{\mathrm{A}}^{\text {tot }} N_{\mathrm{B}}^{\text {tot }}$ pairs. For a given pair, the probability $p_{\mathrm{AB}}$ of being a "molecule" is given by the probability of atom B residing within a volume $V_{\mathrm{ch}}=(4 / 3) \pi \ell_{\mathrm{ch}}^{3}$ of radius $\ell_{\mathrm{ch}}$ around atom $\mathrm{A}$, which is simply given by $p_{\mathrm{AB}}=V_{\mathrm{ch}} / V$ if the total volume of the gas mixture is $V$. The expectation value of the number of molecules is thus equal to 
$N_{\mathrm{AB}}=p_{\mathrm{AB}} N_{\mathrm{A}}^{\text {tot }} N_{\mathrm{B}}^{\text {tot }}$ and the corresponding equilibrium concentration

$$
c_{\mathrm{AB}}=\frac{N_{\mathrm{AB}}}{V}=c_{\mathrm{A}}^{\mathrm{tot}} c_{\mathrm{B}}^{\mathrm{tot}} V_{\mathrm{ch}}=\frac{c_{\mathrm{A}}^{\mathrm{tot}} c_{\mathrm{B}}^{\mathrm{tot}}}{c_{\mathrm{ch}}},
$$

where we define a "chemical reference concentration" $c_{\mathrm{ch}}=1 / V_{\mathrm{ch}}$, and $c_{\mathrm{A} / \mathrm{B}}^{\mathrm{tot}}=N_{\mathrm{A} / \mathrm{B}}^{\mathrm{tot}} / V$ are the total concentrations of atoms A and B, respectively, counting both the "free" atoms and those that are part of molecules $\mathrm{AB}$ at a given instant of time. The chemical reference concentration can be expressed more comprehensibly as $c_{\mathrm{ch}}=N_{\mathrm{ch}} / V$, where $N_{\mathrm{ch}}=V / V_{\mathrm{ch}}$ is the total number of "chemical volume elements" $V_{\mathrm{ch}}$. We will see below that Eq. (1) is valid up to first order in $\left(c_{\mathrm{A}}^{\text {tot }} / c_{\mathrm{ch}}\right)$ and $\left(c_{\mathrm{B}}^{\text {tot }} / c_{\mathrm{ch}}\right)$. The equilibrium concentrations of free atoms are given by $c_{\mathrm{A}}=c_{\mathrm{A}}^{\text {tot }}-c_{\mathrm{AB}}=c_{\mathrm{A}}^{\text {tot }}\left(1-c_{\mathrm{B}}^{\text {tot }} / c_{\mathrm{ch}}\right)$ and $c_{\mathrm{B}}=c_{\mathrm{B}}^{\text {tot }}-c_{\mathrm{AB}}=c_{\mathrm{B}}^{\text {tot }}\left(1-c_{\mathrm{A}}^{\text {tot }} / c_{\mathrm{ch}}\right)$. We thus find

$$
\begin{aligned}
c_{\mathrm{A}} c_{\mathrm{B}} & =c_{\mathrm{A}}^{\mathrm{tot}} c_{\mathrm{B}}^{\mathrm{tot}}\left(1-\frac{c_{\mathrm{A}}^{\mathrm{tot}}}{c_{\mathrm{ch}}}\right)\left(1-\frac{c_{\mathrm{B}}^{\mathrm{tot}}}{c_{\mathrm{ch}}}\right)=\frac{c_{\mathrm{A}}^{\mathrm{tot}} c_{\mathrm{B}}^{\mathrm{tot}}}{c_{\mathrm{ch}}}\left(c_{\mathrm{ch}}-c_{\mathrm{A}}^{\mathrm{tot}}-c_{\mathrm{B}}^{\mathrm{tot}}+\frac{c_{\mathrm{A}}^{\mathrm{tot}} c_{\mathrm{B}}^{\mathrm{tot}}}{c_{\mathrm{ch}}}\right) \\
& =c_{\mathrm{AB}}\left(c_{\mathrm{ch}}-c_{\mathrm{A}}^{\mathrm{tot}}-c_{\mathrm{B}}^{\mathrm{tot}}+c_{\mathrm{AB}}\right)=c_{\mathrm{AB}}\left(c_{\mathrm{ch}}-c_{\mathrm{A}}-c_{\mathrm{B}}-c_{\mathrm{AB}}\right)=c_{\mathrm{AB}} c_{*},
\end{aligned}
$$

where $c_{*}=c_{\mathrm{ch}}-c_{\mathrm{A}}-c_{\mathrm{B}}-c_{\mathrm{AB}}=\left(N_{\mathrm{ch}}-N_{\mathrm{A}}-N_{\mathrm{B}}-N_{\mathrm{AB}}\right) / V$ is the equilibrium concentration of empty, or unoccupied, chemical volume elements $V_{\mathrm{ch}}$. Note that $N_{\mathrm{A}}$ and $N_{\mathrm{B}}$ refer to the equilibrium numbers of free atoms only, without counting those atoms that are part of molecules. If we think of the $N_{\text {ch }}$ chemical volume elements as the sites of a lattice, we find that Eq. (2) has the same structure as the condition for equilibrium coverages on such lattice. Thus the chemical volume elements $V_{\text {ch }}$ introduce a "chemical partitioning" of space, and the resulting equations are equivalent to a "chemistry on a lattice" description, to be discussed in more detail further below. Importantly, we highlight the natural appearance of the concentration $c_{*}$ of empty, or unoccupied, chemical volume elements in Eq. (2) that balances the units on both sides of the equation. In the dilute limit, we have $c_{*} \approx c_{\mathrm{ch}}$.

Ideal Law of Mass Action. We now generalize our analysis to arbitrary molecular stoichiometry. Considering an ideal gas mixture of a total of $N_{\mathrm{A}}^{\text {tot }}$ atoms of type $\mathrm{A}, N_{\mathrm{B}}^{\text {tot }}$ atoms of type $\mathrm{B}$, and $N_{\mathrm{C}}^{\text {tot }}$ atoms of type $\mathrm{C}$ contained within a volume $V$, we count the number $N_{\mathrm{A}_{\alpha} \mathrm{B}_{\beta} \mathrm{C}_{\gamma}}$ of molecules $\mathrm{A}_{\alpha} \mathrm{B}_{\beta} \mathrm{C}_{\gamma}$ with arbitrary integer stoichiometries $\alpha, \beta, \gamma$. Further generalization to more than three different atom types will be straightforward. Here, we apply the same definition of a molecular entity as before, namely we require that all constituents reside within a volume $V_{\mathrm{ch}}$ characterizing the size of the molecule, while all other atoms of the ideal gas mixture must be outside of the molecular volume $V_{\text {ch }}$. We do not require 
any specific arrangement, or ordering, of the constituents, so our definition of a molecular entity is isotropic. Again, the constituent atoms are assumed to be point-like ideal gas particles that do not interact with each other. Taking into account the indistinguishability of atoms of same type, the total number of $(\alpha, \beta, \gamma)$-tuples of atoms $\mathrm{A}, \mathrm{B}$, and $\mathrm{C}$ in the entire ideal gas mixture is given by the product of binomial coefficients $\left(\begin{array}{c}N_{\alpha}^{\text {tot }} \\ \alpha\end{array}\right)\left(\begin{array}{c}N_{\beta}^{\text {tot }} \\ \beta\end{array}\right)\left(\begin{array}{c}N_{C}^{\text {tot }} \\ \gamma\end{array}\right)$. For each of these tuples, the probability of being counted as a molecule $\mathrm{A}_{\alpha} \mathrm{B}_{\beta} \mathrm{C}_{\gamma}$ is given by two factors: First, the probability of finding $(\alpha+\beta+\gamma-1)$ of the constituents within a critical distance $\ell_{\mathrm{ch}}$, i.e. within a volume $V_{\mathrm{ch}}$, around an arbitrarily chosen "first" constituent, which is equal to $\left(V_{\mathrm{ch}} / V\right)^{\alpha+\beta+\gamma-1}$. And second, the probability of finding none of the $N_{\mathrm{A}}^{\text {tot }}+N_{\mathrm{B}}^{\text {tot }}+N_{\mathrm{C}}^{\text {tot }}-(\alpha+\beta+\gamma)$ other atoms of the gas mixture within that same volume element $V_{\text {ch }}$, which is equal to $\left(1-V_{\mathrm{ch}} / V\right)^{N_{\mathrm{A}}^{\text {tot }}+N_{\mathrm{B}}^{\text {tot }}+N_{\mathrm{C}}^{\text {tot }}-(\alpha+\beta+\gamma)}$. We thus obtain

$$
\begin{aligned}
N_{\mathrm{A}_{\alpha} \mathrm{B}_{\beta} \mathrm{C}_{\gamma}} & =\left(\begin{array}{c}
N_{\mathrm{A}}^{\mathrm{tot}} \\
\alpha
\end{array}\right)\left(\begin{array}{c}
N_{\mathrm{B}}^{\mathrm{tot}} \\
\beta
\end{array}\right)\left(\begin{array}{c}
N_{\mathrm{C}}^{\mathrm{tot}} \\
\gamma
\end{array}\right)\left(\frac{V_{\mathrm{ch}}}{V}\right)^{\alpha+\beta+\gamma-1}\left(1-\frac{V_{\mathrm{ch}}}{V}\right)^{N_{\mathrm{A}}^{\mathrm{tot}}+N_{\mathrm{B}}^{\mathrm{tot}}+N_{\mathrm{C}}^{\mathrm{tot}}-(\alpha+\beta+\gamma)} \\
& \stackrel{\mathrm{TL}}{=} \frac{1}{\alpha ! \beta ! \gamma !} \frac{\left(N_{\mathrm{A}}^{\mathrm{tot}}\right)^{\alpha}\left(N_{\mathrm{B}}^{\mathrm{tot}}\right)^{\beta}\left(N_{\mathrm{C}}^{\mathrm{tot}}\right)^{\gamma}}{\left(N_{\mathrm{ch}}\right)^{\alpha+\beta+\gamma-1}}\left(1-\frac{V_{\mathrm{ch}}}{V}\right)^{N_{\mathrm{A}}^{\mathrm{tot}}+N_{\mathrm{B}}^{\mathrm{tot}}+N_{\mathrm{C}}^{\mathrm{tot}}-(\alpha+\beta+\gamma)}
\end{aligned}
$$

where we used $\left(\begin{array}{c}N_{\alpha}^{\text {tot }} \\ \alpha\end{array}\right) \stackrel{\mathrm{TL}}{\longrightarrow}\left(N_{\mathrm{A}}^{\mathrm{tot}}\right)^{\alpha} / \alpha$ ! in the thermodynamic limit (TL), and likewise for $\mathrm{B}$ and $\mathrm{C}$, and we inserted the definition of the total number of chemical volume elements $N_{\text {ch }}=V / V_{\text {ch }}$. It is interesting to note that we also have $\left(1-V_{\text {ch }} / V\right)^{N_{\mathrm{A}}^{\text {tot }}+N_{\mathrm{B}}^{\text {tot }}+N_{\mathrm{C}}^{\text {tot }}-(\alpha+\beta+\gamma)} \stackrel{\mathrm{TL}}{\longrightarrow}$ $\exp \left(-c_{\text {tot }} / c_{\mathrm{ch}}\right)$, where $c_{\text {tot }}=c_{\mathrm{A}}^{\text {tot }}+c_{\mathrm{B}}^{\text {tot }}+c_{\mathrm{C}}^{\text {tot }}$ is the combined total atom concentration of all species, and $c_{\mathrm{ch}}=N_{\mathrm{ch}} / V$, as before. This "colligative" factor $e^{-\left(c_{\mathrm{tot}} / c_{\mathrm{ch}}\right)}$ will frequently appear throughout the following, because it is equal to the probability of all but a certain set of atoms residing outside a given chemical volume element. Dividing Eq. (4) by the volume $V$, we thus find the equilibrium concentration $c_{\mathrm{A}_{\alpha} \mathrm{B}_{\beta} \mathrm{C}_{\gamma}}$ of molecules $\mathrm{A}_{\alpha} \mathrm{B}_{\beta} \mathrm{C}_{\gamma}$

$$
c_{\mathrm{A}_{\alpha} \mathrm{B}_{\beta} \mathrm{C}_{\gamma}}=\frac{1}{\alpha ! \beta ! \gamma !} \frac{\left(c_{\mathrm{A}}^{\mathrm{tot}}\right)^{\alpha}\left(c_{\mathrm{B}}^{\mathrm{tot}}\right)^{\beta}\left(c_{\mathrm{C}}^{\mathrm{tot}}\right)^{\gamma}}{\left(c_{\mathrm{ch}}\right)^{\alpha+\beta+\gamma-1}} e^{-\left(c_{\mathrm{tot}} / c_{\mathrm{ch}}\right)} .
$$

We note that for the case $\alpha=\beta=1$ and $\gamma=0$, we recover Eq. (1) but with the additional colligative factor $e^{-\left(c_{\mathrm{tot}} / c_{\mathrm{ch}}\right)}$. Importantly, the molecule numbers according to Eq. (3) fulfill the requirement of yielding the correct total atom numbers,

$$
\sum_{\alpha=0}^{N_{\mathrm{A}}^{\text {tot }}} \sum_{\beta=0}^{N_{\mathrm{B}}^{\text {tot }}} \sum_{\gamma=0}^{N_{\mathrm{C}}^{\mathrm{tot}}} \alpha N_{\mathrm{A}_{\alpha} \mathrm{B}_{\beta} \mathrm{C}_{\gamma}}=N_{\mathrm{A}}^{\mathrm{tot}}
$$

and likewise for $N_{\mathrm{B}}^{\text {tot }}$ and $N_{\mathrm{C}}^{\text {tot }}$, which can be readily verified using the binomial theorem. 
Equation (5) relates the actual molecular concentration to the total atomic concentrations. We now seek an analogous form involving the actual concentrations of free atoms instead. First, using $\alpha=\beta=\gamma=0$ in Eq. (4), we find the number $N_{*}=N_{\text {ch }}(1-$ $\left.V_{\mathrm{ch}} / V\right)^{N_{\mathrm{A}}^{\mathrm{tot}}+N_{\mathrm{B}}^{\mathrm{tot}}+N_{\mathrm{C}}^{\mathrm{tot}}}$ of empty, or unoccupied, chemical volume elements. Furthermore, we use the respective numbers of free atoms $N_{\mathrm{A}}=N_{\mathrm{A}}^{\text {tot }}\left(1-V_{\mathrm{ch}} / V\right)^{N_{\mathrm{A}}^{\text {tot }}+N_{\mathrm{B}}^{\text {tot }}+N_{\mathrm{C}}^{\text {tot }}-1}$, and likewise for $N_{\mathrm{B}}$ and $N_{\mathrm{C}}$, to find

$$
N_{\mathrm{A}_{\alpha} \mathrm{B}_{\beta} \mathrm{C}_{\gamma}}=\frac{1}{\alpha ! \beta ! \gamma !} \frac{\left(N_{\mathrm{A}}\right)^{\alpha}\left(N_{\mathrm{B}}\right)^{\beta}\left(N_{\mathrm{C}}\right)^{\gamma}}{\left(N_{*}\right)^{\alpha+\beta+\gamma-1}}
$$

and

$$
c_{\mathrm{A}_{\alpha} \mathrm{B}_{\beta} \mathrm{C}_{\gamma}}=\frac{1}{\alpha ! \beta ! \gamma !} \frac{\left(c_{\mathrm{A}}\right)^{\alpha}\left(c_{\mathrm{B}}\right)^{\beta}\left(c_{\mathrm{C}}\right)^{\gamma}}{\left(c_{*}\right)^{\alpha+\beta+\gamma-1}} .
$$

This relation is of central importance for our further analysis. It has a particularly comprehensible form that relates the equilibrium concentration of a certain molecular species to the stoichiometric product of the equilibrium concentrations of the corresponding free atomic constituents. The number of concentration factors on both sides of the equation is balanced by an appropriate power of the equilibrium concentration $c_{*}=c_{\mathrm{ch}} e^{-\left(c_{\mathrm{tot}} / c_{\mathrm{ch}}\right)}$ of unoccupied chemical volume elements. We note that for the case $\alpha=\beta=1$ and $\gamma=0$, we precisely obtain Eq. (2).

Equation (8) can be regarded as the equilibrium condition for the reaction $\alpha \mathrm{A}+\beta \mathrm{B}+$ $\gamma \mathrm{C} \rightleftharpoons \mathrm{A}_{\alpha} \mathrm{B}_{\beta} \mathrm{C}_{\gamma}$ in an ideal gas mixture. For a general reaction

$$
\sum_{i} \rho_{i} \mathrm{R}_{i} \rightleftharpoons \sum_{j} \pi_{j} \mathrm{P}_{j}
$$

with reactants $\mathrm{R}_{i}=\left(\mathrm{A}_{\alpha_{i}} \mathrm{~B}_{\beta_{i}} \mathrm{C}_{\gamma_{i}}\right)$ and products $\mathrm{P}_{j}=\left(\mathrm{A}_{\alpha_{j}} \mathrm{~B}_{\beta_{j}} \mathrm{C}_{\gamma_{j}}\right)$ an analogous equilibrium condition directly derives from Eq. (8),

$$
\left[\prod_{i}\left(g_{\mathrm{R}_{i}}\right)^{\rho_{i}}\left(c_{\mathrm{R}_{i}}\right)^{\rho_{i}}\right]\left(c_{*}\right)^{\sum_{j} \pi_{j}}=\left[\prod_{j}\left(g_{\mathrm{P}_{j}}\right)^{\pi_{j}}\left(c_{\mathrm{P}_{j}}\right)^{\pi_{j}}\right]\left(c_{*}\right)^{\sum_{i} \rho_{i}},
$$

where $c_{\mathrm{R}_{i}}$ and $c_{\mathrm{P}_{j}}$ are the concentrations of reactants and products, respectively, and $g_{\mathrm{R}_{i}}=$ $\left(\alpha_{i} ! \beta_{i} ! \gamma_{i} !\right)$ and $g_{\mathrm{P}_{j}}=\left(\alpha_{j} ! \beta_{j} ! \gamma_{j} !\right)$ are stoichiometry-dependent combinatorial factors for each of the reactants and products. Importantly, the number of concentration factors on both sides of the equation is balanced by appropriate powers of the equilibrium concentration 
$c_{*}$ of unoccupied chemical volume elements. Equation (10) represents an ideal law of mass action and it can be written in the form

$$
\frac{\prod_{j}\left(c_{\mathrm{P}_{j}}\right)^{\pi_{j}}}{\prod_{i}\left(c_{\mathrm{R}_{i}}\right)^{\rho_{i}}}=K_{\mathrm{id}}
$$

with an ideal equilibrium constant

$$
K_{\mathrm{id}}=g_{\mathrm{r}}\left(c_{*}\right)^{\sum_{j} \pi_{j}-\sum_{i} \rho_{i}}
$$

being equal to the concentration $c_{*}$ of empty chemical volume elements raised to the power of the stoichiometry imbalance between both sides of the reaction and multiplied with an overall combinatorial factor of the reaction $g_{\mathrm{r}}=\prod_{i}\left(g_{\mathrm{R}_{i}}\right)^{\rho_{i}} / \prod_{j}\left(g_{\mathrm{P}_{j}}\right)^{\pi_{j}}$. We emphasize that all of the above was derived entirely and solely from the straightforward distance-based definition of a "molecular entity", which is in line with IUPAC terminology.

\section{B. Chemistry in Ideal Gases: Kinetics}

Having established the ideal equilibrium conditions for the concentration of molecules in ideal gas mixtures, we turn to the ideal kinetics of association and dissociation, i.e. the rates of formation and decay of such molecules. As before, we start with the case of a biatomic molecule $\mathrm{AB}$ formed in an elementary reaction

$$
\mathrm{A}+\mathrm{B} \underset{R_{\mathrm{d}}}{\stackrel{R_{\mathrm{a}}}{\rightleftarrows}} \mathrm{AB}
$$

In a non-interacting gas mixture, molecules $\mathrm{AB}$ will only live for the time of the constituent atoms' fly-by, i.e. for the time the constituents are closer than $\ell_{\mathrm{ch}}$ from each other. Since the atoms $\mathrm{A}$ and $\mathrm{B}$ do not interact, their trajectories are straight lines. Accordingly, also their relative trajectory is a straight line characterized by a certain distance of closest approach $r_{\mathrm{cl}}$ in the $x y$-projection plane perpendicular to their relative trajectory, see Figure 1a. For a given $r_{\mathrm{cl}}$, the lifetime of the molecule $\tau\left(r_{\mathrm{cl}}\right)=\ell\left(r_{\mathrm{cl}}\right) / v_{\mathrm{th}}$ is simply given by the length of the trajectory segment $\ell\left(r_{\mathrm{cl}}\right)=2 \sqrt{\ell_{\mathrm{ch}}^{2}-r_{\mathrm{cl}}^{2}}$ inside the "molecular volume", i.e. inside the sphere with radius $\ell_{\mathrm{ch}}$, divided by the average relative thermal velocity $v_{\text {th }}$ of atoms $\mathrm{A}$ and B. Since any relative $x y$-position in the projected plane is equally probable, we can simply average $\tau\left(r_{\mathrm{cl}}\right)$ over the circular molecular cross-section to find the average lifetime of an $\mathrm{AB}$ molecule

$$
\tau=\frac{2 \pi}{\sigma} \int_{0}^{\ell_{\mathrm{ch}}} \frac{\ell\left(r_{\mathrm{cl}}\right)}{v_{\mathrm{th}}} r_{\mathrm{cl}} \mathrm{d} r_{\mathrm{cl}}=\frac{4}{3} \frac{\ell_{\mathrm{ch}}}{v_{\mathrm{th}}}
$$


where we used polar coordinates and $\sigma=\pi \ell_{\mathrm{ch}}^{2}$ is the cross-section area. Accordingly, the molecular dissociation rate $R_{\mathrm{d}}$ per volume is given by the concentration of $\mathrm{AB}$ divided by the average lifetime,

$$
R_{\mathrm{d}}=\frac{c_{\mathrm{AB}}}{\tau} e^{-\frac{c_{\mathrm{tot}}}{c_{\mathrm{ch}}}}=c_{\mathrm{AB}}\left(\frac{c_{*}}{c_{\mathrm{ch}}}\right) k_{\mathrm{d}}=c_{\mathrm{ch}}\left(\frac{c_{\mathrm{AB}}}{c_{\mathrm{ch}}}\right)\left(\frac{c_{*}}{c_{\mathrm{ch}}}\right) k_{\mathrm{d}},
$$

where we used $e^{-\left(c_{\mathrm{tot}} / c_{\mathrm{ch}}\right)}=c_{*} / c_{\mathrm{ch}}$ and introduced the dissociation rate constant $k_{\mathrm{d}}=1 / \tau=$ $(3 / 4) v_{\mathrm{th}} / \ell_{\mathrm{ch}}$. The additional factor $e^{-\left(c_{\mathrm{tot}} / c_{\mathrm{ch}}\right)}$ appears in Eq. (15) for the following reason: A dissociation event according to the reverse direction of reaction (13) requires both of the resulting atoms $\mathrm{A}$ and $\mathrm{B}$ to be free, meaning that no other atom resides within a chemical volume element $V_{\mathrm{ch}}$ around them. This produces an extra factor $e^{-\left(c_{\mathrm{tot}} / c_{\mathrm{ch}}\right)}$, in addition to the one that is implicitly contained in the concentration $c_{\mathrm{AB}}$. Note that $e^{-\left(c_{\mathrm{tot}} / c_{\mathrm{ch}}\right)} \approx 1$ for dilute gas mixtures, so that $R_{\mathrm{d}} \approx c_{\mathrm{AB}} k_{\mathrm{d}}$, as expected. In the last step of Eq. (15), we wrote the concentration factors $c_{\mathrm{AB}}$ and $c_{*}$ as fractions of the chemical reference concentration $c_{\mathrm{ch}}$. We will see later on that this form can be conveniently generalized.

The rate of formation of $\mathrm{AB}$ molecules is directly obtained from the collision rate of atoms A and B, whereby "collision" is simply meant to be the instant in time at which the distance between $\mathrm{A}$ and $\mathrm{B}$ becomes equal to $\ell_{\mathrm{ch}}$. Note that both atoms' trajectories remain entirely unchanged in the ideal gas scenario. Any such "collision" results in the formation of one molecule, because, due to the unchanged relative trajectory, the distance between the involved atoms will necessarily become smaller than $\ell_{\mathrm{ch}}$ after the collision event, thus fulfilling our definition of a molecular entity. Hence, the $\mathrm{AB}$ association rate $R_{\mathrm{a}}$ is equal to the two-body collision rate in an ideal gas mixture, which, using the collision cross section $\sigma=\pi \ell_{\mathrm{ch}}^{2}$, is obtained from a standard textbook exercise, $R_{\mathrm{a}}=c_{\mathrm{A}} c_{\mathrm{B}} v_{\mathrm{th}} \sigma=c_{\mathrm{A}} c_{\mathrm{B}} k_{\text {coll }}^{(2)}$, where $k_{\text {coll }}^{(2)}=v_{\text {th }} \sigma$ is the corresponding rate constant of collision theory.

We will now redefine the rate constant in order to make the symmetry between the forward and backward reaction rates visible. Because $V_{\mathrm{ch}}=(4 / 3) \pi \ell_{\mathrm{ch}}^{3}=1 / c_{\mathrm{ch}}$, we can write the two-body collision cross section in the form $\sigma=\pi \ell_{\mathrm{ch}}^{2}=(3 / 4) V_{\mathrm{ch}} / \ell_{\mathrm{ch}}=3 /\left(4 \ell_{\mathrm{ch}} c_{\mathrm{ch}}\right)$ to obtain

$$
R_{\mathrm{a}}=c_{\mathrm{A}} c_{\mathrm{B}} v_{\mathrm{th}} \sigma=\frac{c_{\mathrm{A}} c_{\mathrm{B}}}{c_{\mathrm{ch}}}\left(\frac{3}{4} \frac{v_{\mathrm{th}}}{\ell_{\mathrm{ch}}}\right)=c_{\mathrm{ch}}\left(\frac{c_{\mathrm{A}}}{c_{\mathrm{ch}}}\right)\left(\frac{c_{\mathrm{B}}}{c_{\mathrm{ch}}}\right) k_{\mathrm{a}},
$$

where we introduced the association rate constant $k_{\mathrm{a}}=(3 / 4) v_{\mathrm{th}} / \ell_{\mathrm{ch}}$, which is related to the typically used two-body collision rate constant by $k_{\mathrm{a}}=c_{\mathrm{ch}} k_{\text {coll }}^{(2)}$. We note that the association 
rate constant is precisely equal to the dissociation rate constant introduced in Eq. (15), $k_{\mathrm{a}}=k_{\mathrm{d}}$. This allows us to describe the association and dissociation rates of molecules $\mathrm{AB}$ in an ideal gas mixture by the very same ideal rate constant $k_{\text {id }}$ being the inverse of the molecular lifetime,

$$
k_{\mathrm{id}}=\frac{3}{4} \frac{v_{\mathrm{th}}}{\ell_{\mathrm{ch}}} .
$$

Importantly, the equality of the rate constants $k_{\mathrm{a}}=k_{\mathrm{d}}=k_{\mathrm{id}}$ guarantees that the association and dissociation rates, Eqs. (16) and (15), fulfill the condition of detailed balance, or microscopic reversibility. At equilibrium, characterized by Eq. (8), we have $c_{\mathrm{AB}} c_{*}=c_{\mathrm{A}} c_{\mathrm{B}}$, from which the dynamic equilibrium $R_{\mathrm{a}}=R_{\mathrm{d}}$ of reaction (13) follows. The value of the ideal rate constant $k_{\text {id }}$ depends on the choice of the critical distance $\ell_{\mathrm{ch}}$. Furthermore, via the relative thermal velocity $v_{\mathrm{th}}=\sqrt{8 k_{\mathrm{B}} T / \pi \mu}$, it depends on the reduced mass $\mu=m_{\mathrm{A}} m_{\mathrm{B}} /\left(m_{\mathrm{A}}+m_{\mathrm{B}}\right)$ of the atom pair, and it is proportional to the square root of the temperature $T$. For $\ell_{\mathrm{ch}}$ of the order of few $\AA$ and a typical thermal velocity at room temperature of the order of $100-1000 \mathrm{~m} / \mathrm{s}$, we have $k_{\mathrm{id}} \approx 10^{12}-10^{13} \mathrm{~s}^{-1}$. For comparison, the corresponding conventional two-body collision rate constant has a value $k_{\text {coll }}^{(2)} \approx 10^{-10} \mathrm{~s}^{-1} \mathrm{~cm}^{3}$, different both in magnitude and units.

Of apples and oranges. For elementary reactions of type (13) in ideal gas mixtures, we found identical rate constants for the forward and backward reaction after renormalizing the two-body collision rate constant $k_{\text {coll }}^{(2)}$ by extracting a factor $1 / c_{\mathrm{ch}}$. This is remarkable, because conventionally one would use the second-order rate constant $k_{\mathrm{f}}^{(2)}=k_{\text {coll }}^{(2)}$ to describe the bimolecular forward reaction and the first-order rate constant $k_{\mathrm{b}}^{(1)}=k_{\mathrm{d}}$ for the unimolecular backward direction. Obviously, such conventional rate constants for different reaction orders are like "apples and oranges" and cannot be compared, neither in magnitude nor in units. Our approach enables a consistent "apples-to-apples" comparison of rate constants for different reaction orders. Furthermore, conventional second-order rate constants describing bimolecular reactions are dependent on the reference concentration chosen for the concentration factors of the rate equation. Our renormalization scheme yields effective first-order rate constants that are independent of such choice. These aspects will be discussed again in the context of the excess rate constants further below.

Elementary reactions of molecularity $\geq 3$ do not occur in ideal gas mixtures. According to our definition, a termolecular elementary reaction $\mathrm{A}+\mathrm{B}+\mathrm{C} \rightarrow \mathrm{ABC}$ would correspond 
to a three-body collision event when at least two of the pairs $\mathrm{A}-\mathrm{B}, \mathrm{A}-\mathrm{C}$, and $\mathrm{B}-\mathrm{C}$ have a distance precisely equal to $\ell_{\mathrm{ch}}$. This is equivalent to two two-body collisions happening at the very same instant in time, the probability of which is zero. Therefore, in ideal gas mixtures, a reaction $\mathrm{A}+\mathrm{B}+\mathrm{C} \rightarrow \mathrm{ABC}$ necessarily proceeds in two bimolecular steps, e.g. $\mathrm{A}+\mathrm{B}+\mathrm{C} \rightarrow \mathrm{AB}+\mathrm{C} \rightarrow \mathrm{ABC}$. The ideal kinetics of bimolecular reactions of type (13) are therefore sufficient to describe any reaction in ideal gas mixtures.

\section{Reaction Barriers, Transition States, and Binding Energy}

Having discussed equilibrium and kinetics of ideal gas reactions, we take a step towards a more realistic model by including the possibility of an energetic barrier between reactants and products, and a binding energy in the molecular state. We will adopt Eyring's statistical treatment $^{2}$ and demonstrate that our ideal gas framework smoothly couples to transition state theory (TST). Again, we consider a bimolecular elementary reaction according to Eq. (13). As before, we consider point-like atoms and define a molecule AB as an atom pair where atom $B$ is located within a sphere of radius $\ell_{\text {ch }}$ around atom $A$. We now include the possibility of a change in potential energy to a binding energy $E_{\mathrm{AB}} \leq 0$ in the molecular state, where the potential energy zero refers to the free atomic state. Furthermore, we include a transition state (TS), or activated complex, with potential energy $E_{\mathrm{TS}} \geq 0$ that corresponds to a spherical shell of width $\delta_{\mathrm{TS}} \ll \ell_{\mathrm{ch}}$ around the spherical molecular volume $V_{\text {ch }}$ in the configuration space of the atom pair, as shown in Figure 1b. This simple transition state model is isotropic and the reaction coordinate is the distance $r$ between atoms $\mathrm{A}$ and B. The potential energy along the reaction coordinate thus reads

$$
E(r)= \begin{cases}E_{\mathrm{AB}} & \text { for } r \leq \ell_{\mathrm{ch}} \\ E_{\mathrm{TS}} & \text { for } \ell_{\mathrm{ch}}<r \leq \ell_{\mathrm{ch}}+\delta_{\mathrm{TS}} \\ 0 & \text { for } \ell_{\mathrm{ch}}+\delta_{\mathrm{TS}}<r\end{cases}
$$

which is shown in Figure 1c. Although highly simplified, this isotropic TST (iTST) model contains the essential features of TST, namely a distinct part of the system phase space representing the summit region of the potential energy barrier that separates product states from reactant states. In fact, Eyring ${ }^{2}$ used a very similar model to demonstrate how his TST rate expression reduces to the one from collision theory. Following Eyring's approach, we write the rate of the forward (association) direction of reaction (13) as the decay rate of 
the TS towards the product $\mathrm{AB}$, given by

$$
R_{\mathrm{a}}^{\mathrm{iTST}}=\frac{N_{\mathrm{A}} N_{\mathrm{B}}}{V} \frac{1}{2}\left(\frac{Z_{\mathrm{TS}}^{(1)}}{Z_{\mathrm{A}}^{(1)} Z_{\mathrm{B}}^{(1)}}\right) \frac{\left\langle\left|v_{r}\right|\right\rangle_{\mathrm{th}}}{\delta_{\mathrm{TS}}},
$$

where $\left\langle\left|v_{r}\right|\right\rangle_{\text {th }}$ is the average magnitude of the relative thermal velocity of $\mathrm{A}$ and $\mathrm{B}$ in the $r a-$ dial direction along the reaction coordinate. The factor $(1 / 2)$ accounts for the fact that only half of the activated complexes move towards $\mathrm{AB}$ association along the reaction coordinate (the other half moving towards dissociation).

$$
Z_{\mathrm{A}}^{(1)}=V_{*}\left(2 \pi m_{\mathrm{A}} k_{\mathrm{B}} T / h^{2}\right)^{3 / 2}
$$

and

$$
Z_{\mathrm{B}}^{(1)}=V_{*}\left(2 \pi m_{\mathrm{B}} k_{\mathrm{B}} T / h^{2}\right)^{3 / 2}
$$

are the ideal gas partition functions per free atom of $\mathrm{A}$ and $\mathrm{B}$, respectively. Here, $V_{*}=$ $N_{*} V_{\mathrm{ch}}=V e^{-\left(c_{\mathrm{tot}} / c_{\mathrm{ch}}\right)}$ is the empty volume accessible for free motion that is slightly reduced compared to the total volume $V$ by the colligative factor $e^{-\left(c_{\mathrm{tot} t} / c_{\mathrm{ch}}\right)}$. The TS partition function

$$
Z_{\mathrm{TS}}^{(1)}=\left(\frac{2 \pi\left[m_{\mathrm{A}}+m_{\mathrm{B}}\right] k_{\mathrm{B}} T}{h^{2}}\right)^{3 / 2}\left(\frac{2 \pi \mu k_{\mathrm{B}} T}{h^{2}}\right)^{3 / 2} V_{*} V_{\mathrm{TS}} e^{-\frac{c_{\mathrm{tot}}}{c_{\mathrm{ch}}}} e^{-\frac{E_{\mathrm{TS}}}{k_{\mathrm{B}} T}}
$$

can be split into the center of mass motion and the relative motion of the TS constituents $\mathrm{A}$ and $\mathrm{B}$ with reduced mass $\mu$. The latter is confined to the TS volume $V_{\mathrm{TS}}$ and it carries a Boltzmann factor $e^{-E_{\mathrm{TS}} / k_{\mathrm{B}} T}$ of the TS potential energy. An additional factor $e^{-\left(c_{\mathrm{tot}} / c_{\mathrm{ch}}\right)}$ appears in Eq. (22) for the following reason: The free atom partition functions, Eqs. (20) and (21), both implicitly contain such a factor as part of the empty accessible volume $V_{*}=$ $V e^{-\left(c_{\mathrm{tot}} / c_{\mathrm{ch}}\right)}$. This stems from the fact that each free atom defines an exclusion volume $V_{\mathrm{ch}}$ where no other atoms must be present. In the transition state partition function, Eq. (22), one such factor is implicitly retained in $V_{*}$. However, we also need to preserve the second factor, because the special atomic configuration of the transition state defines an exclusion volume of $\approx 2 V_{\text {ch }}$. Only in the product state $\mathrm{AB}$, the total exclusion volume gets contracted to $\approx V_{\mathrm{ch}}$, for which reason the $\mathrm{AB}$ partition function given in Eq. (25) below does not contain the additional factor $e^{-\left(c_{\mathrm{tot}} / c_{\mathrm{ch}}\right)}$.

Since $\delta_{\mathrm{TS}} \ll \ell_{\mathrm{ch}}$, the volume of the spherical TS shell of width $\delta_{\mathrm{TS}}$ and radius $\ell_{\mathrm{ch}}$ is well approximated by $V_{\mathrm{TS}}=4 \pi \ell_{\mathrm{ch}}^{2} \delta_{\mathrm{TS}}=3 \delta_{\mathrm{TS}} / \ell_{\mathrm{ch}} c_{\mathrm{ch}}$, where we used $V_{\mathrm{ch}}=(4 / 3) \pi \ell_{\mathrm{ch}}^{3}=1 / c_{\mathrm{ch}}$. 
Furthermore, it is shown in the Supplementary Material that $\left\langle\left|v_{r}\right|\right\rangle_{\mathrm{th}}=v_{\mathrm{th}} / 2=\sqrt{2 k_{\mathrm{B}} T / \pi \mu}$, where $v_{\text {th }}$ is the same as in Eqs. (16) and (17). The kinetic energy factors in the quotient of the partition functions in Eq. (19) exactly cancel, so that the association rate reads

$$
\begin{aligned}
R_{\mathrm{a}}^{\mathrm{iTST}} & =\frac{N_{\mathrm{A}} N_{\mathrm{B}}}{V} \frac{V_{\mathrm{TS}}}{2 V_{*}} e^{-\frac{c_{\mathrm{cot}}}{c_{\mathrm{ch}}}} e^{-\frac{E_{\mathrm{TS}}}{k_{\mathrm{B}} T}} \frac{v_{\mathrm{th}}}{2 \delta_{\mathrm{TS}}}=\frac{c_{\mathrm{A}} c_{\mathrm{B}}}{c_{\mathrm{ch}}}\left(\frac{3}{4} \frac{v_{\mathrm{th}}}{\ell_{\mathrm{ch}}}\right) e^{-\frac{E_{\mathrm{TS}}}{k_{\mathrm{B}} T}} \\
& =\frac{c_{\mathrm{A}} c_{\mathrm{B}}}{c_{\mathrm{ch}}}\left(k_{\mathrm{id}} e^{-\frac{E_{\mathrm{TS}}}{k_{\mathrm{B}} T}}\right)=c_{\mathrm{ch}}\left(\frac{c_{\mathrm{A}}}{c_{\mathrm{ch}}}\right)\left(\frac{c_{\mathrm{B}}}{c_{\mathrm{ch}}}\right) k_{\mathrm{a}}^{\mathrm{iTST}},
\end{aligned}
$$

where we used $V_{*}=V e^{-\left(c_{\mathrm{tot}} / c_{\mathrm{ch}}\right)}$. The association rate of the isotropic TST model is equal to the ideal association rate according to Eq. (16) multiplied by an Arrhenius factor $e^{-E_{\mathrm{TS}} / k_{\mathrm{B}} T}$ with an activation energy $E_{\mathrm{TS}}$ equal to the potential energy difference between the transition state and the free atomic reactant states. This is consistent with collision theory where the presence of an activation barrier of height $E_{\mathrm{TS}}$ reduces the reaction rate by such an Arrhenius factor. Importantly, we again find that the association rate of the isotropic TST (iTST) model can be described by a renormalized first-order rate constant $k_{\mathrm{a}}^{\mathrm{iTST}}=k_{\mathrm{id}} e^{-E_{\mathrm{TS}} / k_{\mathrm{B}} T}$ being the ideal rate constant $k_{\text {id }}$ of Eq. (17) multiplied by the Arrhenius factor $e^{-E_{\mathrm{TS}} / k_{\mathrm{B}} T}$.

The isotropic TST model further enables the computation of the rate of the backward (dissociation) direction of reaction (13) as the decay rate of the TS towards the free reactant atoms A and B. Analogous to Eq. (19), it is given by

$$
R_{\mathrm{d}}^{\mathrm{iTST}}=\frac{N_{\mathrm{AB}}}{V} \frac{1}{2}\left(\frac{Z_{\mathrm{TS}}^{(1)}}{Z_{\mathrm{AB}}^{(1)}}\right) \frac{\left\langle\left|v_{r}\right|\right\rangle_{\mathrm{th}}}{\delta_{\mathrm{TS}}} .
$$

In this case, we assume the TS to be equilibrated with the molecular $\mathrm{AB}$ states. The partition function per molecule of $\mathrm{AB}$ is given by

$$
Z_{\mathrm{AB}}^{(1)}=\left(\frac{2 \pi\left[m_{\mathrm{A}}+m_{\mathrm{B}}\right] k_{\mathrm{B}} T}{h^{2}}\right)^{3 / 2}\left(\frac{2 \pi \mu k_{\mathrm{B}} T}{h^{2}}\right)^{3 / 2} V_{*} V_{\mathrm{ch}} e^{-\frac{E_{\mathrm{AB}}}{k_{\mathrm{B}} T}},
$$

where we used the fact that the molecular volume $V_{\mathrm{AB}}$ is, by definition, equal to the chemical volume element, $V_{\mathrm{AB}}=V_{\mathrm{ch}}$. Inserting Eqs. (22) and (25) into Eq. (24), we obtain

$$
\begin{aligned}
R_{\mathrm{d}}^{\mathrm{iTST}} & =\frac{N_{\mathrm{AB}}}{V} \frac{V_{\mathrm{TS}}}{2 V_{\mathrm{ch}}} e^{-\frac{c_{\mathrm{cot}}}{c_{\mathrm{ch}}}} e^{-\frac{E_{\mathrm{TS}}-E_{\mathrm{AB}}}{k_{\mathrm{B}} T}} \frac{v_{\mathrm{th}}}{2 \delta_{\mathrm{TS}}}=c_{\mathrm{AB}} e^{-\frac{c_{\mathrm{cot}}}{c_{\mathrm{ch}}}}\left(\frac{3}{4} \frac{v_{\mathrm{th}}}{\ell_{\mathrm{ch}}}\right) e^{-\frac{E_{\mathrm{TSS}}-E_{\mathrm{AB}}}{k_{\mathrm{B}} T}} \\
& =c_{\mathrm{AB}}\left(\frac{c_{*}}{c_{\mathrm{ch}}}\right)\left(k_{\mathrm{id}} e^{-\frac{E_{\mathrm{TS}}-E_{\mathrm{AB}}}{k_{\mathrm{B}} T}}\right)=c_{\mathrm{ch}}\left(\frac{c_{\mathrm{AB}}}{c_{\mathrm{ch}}}\right)\left(\frac{c_{*}}{c_{\mathrm{ch}}}\right) k_{\mathrm{d}}^{\mathrm{iTST}},
\end{aligned}
$$

where we used $V_{\mathrm{TS}} / V_{\mathrm{ch}}=V_{\mathrm{TS}} c_{\mathrm{ch}}=3 \delta_{\mathrm{TS}} / \ell_{\mathrm{ch}}$ and $e^{-\left(c_{\mathrm{tot}} / c_{\mathrm{ch}}\right)}=c_{*} / c_{\mathrm{ch}}$. Also here, we find that the dissociation rate of the isotropic TST model is equal to the ideal dissociation rate 
according to Eq. (15) multiplied by an Arrhenius factor $e^{-\left(E_{\mathrm{TS}}-E_{\mathrm{AB}}\right) / k_{\mathrm{B}} T}$ with an activation energy $\left(E_{\mathrm{TS}}-E_{\mathrm{AB}}\right)$ equal to the potential energy difference between the transition state and the molecular state. The corresponding rate constant $k_{\mathrm{d}}^{\mathrm{iTST}}=k_{\mathrm{id}} e^{-\left(E_{\mathrm{TS}}-E_{\mathrm{AB}}\right) / k_{\mathrm{B}} T}$ is thus given by the ideal rate constant $k_{\text {id }}$ of Eq. (17) multiplied by the Arrhenius factor.

The dynamic equilibrium condition for the isotropic TST model requires the association and dissociation rates of Eqs. (23) and (26), respectively, to be equal. We obtain

$$
c_{\mathrm{AB}} c_{*}=c_{\mathrm{A}} c_{\mathrm{B}} e^{-E_{\mathrm{AB}} / k_{\mathrm{B}} T} .
$$

The equilibrium concentration of AB molecules in the isotropic TST model is given by the ideal expression from Eq. (2) multiplied with a Boltzmann factor $e^{-E_{\mathrm{AB}} / k_{\mathrm{B}} T}$. Since the molecular binding energy $E_{\mathrm{AB}} \leq 0$, the $\mathrm{AB}$ equilibrium concentration of the iTST model is generally larger than the ideal equilibrium concentration, as expected.

We conclude that the isotropic TST model precisely yields the expressions for association/dissociation in ideal gas mixtures, both in terms of equilibrium and kinetics, in the limit $E_{\mathrm{TS}} \rightarrow 0$ and $E_{\mathrm{AB}} \rightarrow 0$ of zero activation and binding energy, respectively. Thus, our distance-based definition of molecules in ideal gas mixtures simply corresponds to the transition state theory limit of an entirely flat potential energy landscape.

\section{Chemical Potentials and Free Energy of Reaction}

We use the partition functions of Eqs. (20), (21), and (25) with $E_{\mathrm{AB}}=0$ to compute the ideal chemical potentials $\mu_{\mathrm{A}}, \mu_{\mathrm{B}}$, and $\mu_{\mathrm{AB}}$ of free atoms $\mathrm{A}, \mathrm{B}$, and ideal molecules $\mathrm{AB}$, respectively. Using the relation $Z^{(N)}=\left(Z^{(1)}\right)^{N} / N$ ! between the $N$-particle and singleparticle partition functions for each of these species, together with the Helmholtz free energy $A=-k_{\mathrm{B}} T \ln Z^{(N)}$ and the Stirling approximation $\ln N ! \approx N \ln N-N$, we obtain $A=$ $-N k_{\mathrm{B}} T\left[\ln \left(Z^{(1)} / N\right)+1\right]$ from which we compute the chemical potential $\mu=(\partial A / \partial N)_{T, V}=$ $-k_{\mathrm{B}} T \ln \left(Z^{(1)} / N\right)$. The single-particle partition functions can be written in the form $Z_{\mathrm{A}}^{(1)}=$

$V_{*} / \lambda_{\mathrm{th}, m_{\mathrm{A}}}^{3}, Z_{\mathrm{B}}^{(1)}=V_{*} / \lambda_{\mathrm{th}, m_{\mathrm{B}}}^{3}$, and $Z_{\mathrm{AB}}^{(1)}=V_{*} V_{\mathrm{ch}} / \lambda_{\mathrm{th}, \mu}^{3} \lambda_{\mathrm{th},\left(m_{\mathrm{A}}+m_{\mathrm{B}}\right)}^{3}$ with the corresponding thermal wavelengths $\lambda_{\mathrm{th}, m}=h /\left(2 \pi m k_{\mathrm{B}} T\right)^{1 / 2}$. Together with $V_{*}=V e^{-\left(c_{\mathrm{tot}} / c_{\mathrm{ch}}\right)}$, we thus find 
the chemical potentials

$$
\begin{aligned}
\mu_{\mathrm{A}} & =k_{\mathrm{B}} T \ln \left(\lambda_{\mathrm{th}, m_{\mathrm{A}}}^{3} c_{\mathrm{A}} e^{\frac{c_{\mathrm{tot}}}{c_{\mathrm{ch}}}}\right), \\
\mu_{\mathrm{B}} & =k_{\mathrm{B}} T \ln \left(\lambda_{\mathrm{th}, m_{\mathrm{B}}}^{3} c_{\mathrm{B}} e^{\frac{c_{\mathrm{tot}}}{c_{\mathrm{ch}}}}\right), \\
\mu_{\mathrm{AB}} & =k_{\mathrm{B}} T \ln \left(\lambda_{\mathrm{th}, \mu}^{3} \lambda_{\mathrm{th},\left(m_{\mathrm{A}}+m_{\mathrm{B}}\right)}^{3} c_{\mathrm{AB}} c_{\mathrm{ch}} e^{\frac{c_{\mathrm{tot}}}{c_{\mathrm{ch}}}}\right),
\end{aligned}
$$

where $V_{\mathrm{ch}}=1 / c_{\mathrm{ch}}$ was used in the last equation.

We can now compute the Gibbs free energy of reaction for the ideal gas association reaction $\mathrm{A}+\mathrm{B} \rightarrow \mathrm{AB}$ for given concentrations $c_{\mathrm{A}}, c_{\mathrm{B}}$, and $c_{\mathrm{AB}}$,

$$
\Delta_{\mathrm{r}} G_{\mathrm{id}}=\mu_{\mathrm{AB}}-\mu_{\mathrm{A}}-\mu_{\mathrm{B}}=k_{\mathrm{B}} T \ln \left(\frac{c_{\mathrm{AB}} c_{\mathrm{ch}}}{c_{\mathrm{A}} c_{\mathrm{B}} e^{\frac{c_{\mathrm{cot}}}{c_{\mathrm{ch}}}}}\right)=k_{\mathrm{B}} T \ln \left(\frac{c_{\mathrm{AB}} c_{*}}{c_{\mathrm{A}} c_{\mathrm{B}}}\right),
$$

where we used $c_{*}=c_{\mathrm{ch}} e^{-\left(c_{\mathrm{tot}} / c_{\mathrm{ch}}\right)}$ in the last step. Note that all thermal wavelength factors get canceled, which can be seen from the definition of the reduced mass $\mu=m_{\mathrm{A}} m_{\mathrm{B}} /\left(m_{\mathrm{A}}+\right.$ $m_{\mathrm{B}}$ ). Eq. (31) has a comprehensible form, and it should be emphasized that the intrinsic appearance of $c_{*}$ makes the equation independent of the definition of a specific reference state. Importantly, Eq. (31) is consistent with the ideal equilibrium condition of Eq. (2), namely $\Delta_{\mathrm{r}} G_{\mathrm{id}}=0 \Leftrightarrow c_{\mathrm{AB}} c_{*}=c_{\mathrm{A}} c_{\mathrm{B}}$.

We can readily generalize these results. The partition function of a single ideal molecule $\mathrm{A}_{\alpha} \mathrm{B}_{\beta} \mathrm{C}_{\gamma}$ reads

$$
Z_{\mathrm{A}_{\alpha} \mathrm{B}_{\beta} \mathrm{C}_{\gamma}}^{(1)}=\frac{1}{\alpha ! \beta ! \gamma !} \frac{V_{*} V_{\mathrm{ch}}^{\alpha+\beta+\gamma-1}}{\lambda_{\mathrm{th}, m_{\mathrm{A}}}^{3 \alpha} \lambda_{\mathrm{th}, m_{\mathrm{B}}}^{3 \beta} \lambda_{\mathrm{th}, m_{\mathrm{C}}}^{3 \gamma}}
$$

from which we obtain the chemical potential

$$
\mu_{\mathrm{A}_{\alpha} \mathrm{B}_{\beta} \mathrm{C}_{\gamma}}=k_{\mathrm{B}} T \ln \left(\alpha ! \beta ! \gamma ! \lambda_{\mathrm{th}, m_{\mathrm{A}}}^{3 \alpha} \lambda_{\mathrm{th}, m_{\mathrm{B}}}^{3 \beta} \lambda_{\mathrm{th}, m_{\mathrm{C}}}^{3 \gamma} c_{\mathrm{A}_{\alpha} \mathrm{B}_{\beta} \mathrm{C}_{\gamma}} c_{\mathrm{ch}}^{\alpha+\beta+\gamma-1} e^{\frac{c_{\mathrm{tot}}}{c_{\mathrm{ch}}}}\right)
$$

For the general reaction given in Eq. (9), the ideal Gibbs free energy of reaction therefore is

$$
\Delta_{\mathrm{r}} G_{\mathrm{id}}=k_{\mathrm{B}} T \ln \left(\frac{\left[\prod_{j}\left(g_{\mathrm{P}_{j}}\right)^{\pi_{j}}\left(c_{\mathrm{P}_{j}}\right)^{\pi_{j}}\right]\left(c_{*}\right)^{\sum_{i} \rho_{i}}}{\left[\prod_{i}\left(g_{\mathrm{R}_{i}}\right)^{\rho_{i}}\left(c_{\mathrm{R}_{i}}\right)^{\rho_{i}}\right]\left(c_{*}\right)^{\sum_{j} \pi_{j}}}\right)=k_{\mathrm{B}} T \ln \left(\frac{\prod_{j}\left(c_{\mathrm{P}_{j}}\right)^{\pi_{j}}}{\prod_{i}\left(c_{\mathrm{R}_{i}}\right)^{\rho_{i}}} \frac{1}{K_{\mathrm{id}}}\right)
$$

with the ideal equilibrium constant $K_{\text {id }}$ of Eq. (12). At equilibrium, $\Delta_{\mathrm{r}} G_{\text {id }}=0$, we recover the ideal law of mass action, Eq. (11). 


\section{DISCUSSION}

\section{A. Ideal Gas Reference for Real Association/Dissociation Reactions}

The theoretical framework of association and dissociation in ideal gas mixtures developed above provides a generalization of the ideal gas reference, commonly used in thermodynamics, to chemical association and dissociation reactions. We therefore extend the respective terminology and denote quantities that are defined relative to the ideal gas reference as excess quantities.

\section{Equilibrium Constant and Reaction Free Energy}

Based on the ideal Eqs. (10)-(12), we define the excess equilibrium constant of a real association/dissociation reaction of type (9)

$$
K_{\mathrm{xs}}=\frac{K_{\mathrm{c}}}{K_{\mathrm{id}}}=\left(\frac{1}{g_{\mathrm{r}}}\right) \frac{\prod_{j}\left(c_{\mathrm{P}_{j}}^{\mathrm{eq}}\right)^{\pi_{j}}}{\prod_{i}\left(c_{\mathrm{R}_{i}}^{\mathrm{eq}}\right)^{\rho_{i}}} \frac{\left(c_{*}\right)^{\sum_{i} \rho_{i}}}{\left(c_{*}\right)^{\sum_{j} \pi_{j}}}
$$

where $K_{\mathrm{c}}=\prod_{j}\left(c_{\mathrm{P}_{j}}^{\mathrm{eq}}\right)^{\pi_{j}} / \prod_{i}\left(c_{\mathrm{R}_{i}}^{\mathrm{eq}}\right)^{\rho_{i}}$ is the corresponding concentration-based equilibrium constant. Note that here the concentrations $c_{\mathrm{R}_{i}}^{\mathrm{eq}}$ and $c_{\mathrm{P}_{j}}^{\mathrm{eq}}$ refer to the real equilibrium concentrations of reactants and products, respectively. Obviously, $K_{\mathrm{xs}}$ is a dimensionless quantity. For the special case $\sum_{i} \rho_{i}=\sum_{j} \pi_{j}$ of balanced stoichiometries on both sides of the reaction, the ideal equilibrium constant $K_{\text {id }}$ simply reduces to the combinatorial factor $g_{\mathrm{r}}$, which typically is of order unity. In such case, the excess equilibrium constant is essentially equal to the concentration-based equilibrium constant. For unbalanced stoichiometries $\sum_{i} \rho_{i} \neq \sum_{j} \pi_{j}$, the number of concentration factors in the numerator and denominator of $K_{\mathrm{xs}}$ is balanced by an appropriate power of $c_{*}$, making $K_{\mathrm{xs}}$ independent of the choice of concentration units, in contrast to $K_{\mathrm{c}}$. Naturally, the excess equilibrium constant of an ideal reaction is equal to one.

For the isotropic TST model of the reaction $\mathrm{A}+\mathrm{B} \rightleftharpoons \mathrm{AB}$, we obtained the equilibrium condition (27) corresponding to a concentration-based equilibrium constant $K_{\mathrm{c}}=$ $c_{\mathrm{AB}} / c_{\mathrm{A}} c_{\mathrm{B}}=\left(1 / c_{*}\right) e^{-E_{\mathrm{AB}} / k_{\mathrm{B}} T}$. The respective excess equilibrium constant $K_{\mathrm{xs}}=c_{\mathrm{AB}} c_{*} / c_{\mathrm{A}} c_{\mathrm{B}}=$ $e^{-E_{\mathrm{AB}} / k_{\mathrm{B}} T}$ is simply equal to the Boltzmann factor of the $\mathrm{AB}$ binding energy. The excess equilibrium constant thus quantifies how well the reactant and product states are energeti- 
cally aligned on the potential energy surface of the system.

For non-equilibrium of a general reaction (9), characterized by a Gibbs free energy of reaction $\Delta_{\mathrm{r}} G$, we define a corresponding excess Gibbs free energy of reaction

$$
\Delta_{\mathrm{r}} G_{\mathrm{xs}}=\Delta_{\mathrm{r}} G-\Delta_{\mathrm{r}} G_{\mathrm{id}}
$$

with the ideal Gibbs free energy of reaction $\Delta_{\mathrm{r}} G_{\text {id }}$ of Eq. (34). We write $\Delta_{\mathrm{r}} G$ in the form

$$
\Delta_{\mathrm{r}} G=\Delta_{\mathrm{r}} G^{\ominus}+k_{\mathrm{B}} T \ln \left(\frac{\prod_{j}\left(a_{\mathrm{P}_{j}}\right)^{\pi_{j}}}{\prod_{i}\left(a_{\mathrm{R}_{i}}\right)^{\rho_{i}}}\right)
$$

with the standard Gibbs free energy $\Delta_{\mathrm{r}} G^{\ominus}=\sum_{j} \pi_{j} \mu_{\mathrm{P}_{j}}^{\ominus}-\sum_{i} \rho_{i} \mu_{\mathrm{R}_{i}}^{\ominus}$ and activities $a_{\mathrm{R}_{i}}$ and $a_{\mathrm{P}_{j}}$ of reactants and products, respectively. The activities are defined relative to the standard state for each species and can be written in the form $a=\gamma_{\mathrm{c}}\left(c / c^{\ominus}\right)$, where $c$ is the respective concentration, $c^{\ominus}$ the concentration at standard state, and $\gamma_{\mathrm{c}}$ the concentrationbased activity coefficient. Using Eq. (34), we obtain the excess Gibbs free energy of reaction in the form

$$
\Delta_{\mathrm{r}} G_{\mathrm{XS}}=\Delta_{\mathrm{r}} G^{\ominus}+k_{\mathrm{B}} T \ln \left(g_{\mathrm{r}}\right)+k_{\mathrm{B}} T \ln \left(\frac{\prod_{j}\left(c_{*} / c_{\mathrm{P}_{j}}^{\ominus}\right)^{\pi_{j}}}{\prod_{i}\left(c_{*} / c_{\mathrm{R}_{i}}^{\ominus}\right)^{\rho_{i}}}\right)+k_{\mathrm{B}} T \ln \left(\Gamma_{\mathrm{c}, \mathrm{r}}\right)
$$

with the combinatorial factor of the reaction $g_{\mathrm{r}}=\prod_{i}\left(g_{\mathrm{R}_{i}}\right)^{\rho_{i}} / \prod_{j}\left(g_{\mathrm{P}_{j}}\right)^{\pi_{j}}$ introduced further above, and the quotient of activity coefficients $\Gamma_{\mathrm{c}, \mathrm{r}}=\prod_{j}\left(\gamma_{\mathrm{c}, \mathrm{P}_{j}}\right)^{\pi_{j}} / \prod_{i}\left(\gamma_{\mathrm{c}, \mathrm{R}_{i}}\right)^{\rho_{i}}$. For a constant temperature, the only term in Eq. (38) that weakly depends on the actual experimental conditions, i.e. concentrations, is the last one involving $\Gamma_{c, r}$. In Eq. (38), we explicitly take into account the possibility that the standard-state concentrations $c^{\ominus}$ can be different for different species, which is the case, e.g., when they are present in different aggregation states at standard conditions. The consequences thereof and the physical relevance of the excess Gibbs free energy of reaction $\Delta_{\mathrm{r}} G_{\mathrm{xS}}$ for electrochemical reactions will be discussed in detail in Part II. of this series. We finally note that, at equilibrium with $\Delta_{\mathrm{r}} G=0$, the excess Gibbs free energy of reaction is directly related to the excess equilibrium constant via

$$
\Delta_{\mathrm{r}} G_{\mathrm{xs}}^{\mathrm{eq}}=-k_{\mathrm{B}} T \ln \left(K_{\mathrm{xs}}\right)
$$

which follows from Eq. (36) with $\Delta_{\mathrm{r}} G=0$, in combination with Eq. (34) where we recognize $K_{\mathrm{xs}}$ in the argument of the logarithm. 
As an example, we discuss the excess equilibrium constant of the water autoprotolysis reaction $\mathrm{H}_{2} \mathrm{O} \rightleftharpoons \mathrm{H}^{+}+\mathrm{OH}^{-}$. The corresponding concentration-based equilibrium constant is $K_{\mathrm{c}}=c_{\mathrm{H}^{+}} c_{\mathrm{OH}^{-}} / c_{\mathrm{H}_{2} \mathrm{O}, \ell}=1.8 \times 10^{-16} \mathrm{~mol} \mathrm{~L}^{-1}$, where we used $K_{\mathrm{w}}=c_{\mathrm{H}^{+}} c_{\mathrm{OH}^{-}}=1.0 \times$ $10^{-14}\left(\mathrm{~mol} \mathrm{~L}^{-1}\right)^{2}$ and the concentration of liquid water ${ }^{3} c_{\mathrm{H}_{2} \mathrm{O}, \ell}=55.34 \mathrm{~mol} \mathrm{~L}^{-1}$ at $T=25^{\circ} \mathrm{C}$. The corresponding excess equilibrium constant is $K_{\mathrm{xs}}=\left(1 / g_{\mathrm{r}}\right)\left(c_{\mathrm{H}^{+}} c_{\mathrm{OH}^{-}}\right) /\left(c_{\mathrm{H}_{2} \mathrm{O}, \ell} c_{*}\right)$. The combinatorial factor of the reaction is $g_{\mathrm{r}}=2$. As discussed in detail in Part II. of this series, it is reasonable for aqueous reactions to choose the chemical reference concentration $c_{\mathrm{ch}}$ in a way that $c_{*}=c_{\mathrm{ch}} e^{-\left(c_{\mathrm{tot}} / c_{\mathrm{ch}}\right)}$ is equal to the concentration of liquid water. This choice corresponds to a value of $\ell_{\mathrm{ch}} \approx 1.5-1.6 \AA$ in the middle between the $\mathrm{O}-\mathrm{H}$ bond length of $\approx 1 \AA$ within a water molecule and the $\mathrm{O} \cdots \mathrm{H}$ hydrogen bond length of $\approx 2 \AA$ between adjacent water molecules. The amount of arbitrariness introduced by the specific choice of $c_{*}=c_{\mathrm{H}_{2} \mathrm{O}, \ell}=55.34 \mathrm{~mol} \mathrm{~L}^{-1}$ is of order unity and unimportant for the present analysis. With this, we obtain $K_{\mathrm{xs}}=\left(1 / g_{\mathrm{r}}\right)\left(c_{\mathrm{H}^{+}} c_{\mathrm{OH}^{-}}\right) /\left(c_{\mathrm{H}_{2} \mathrm{O}, \ell}\right)^{2}=1.6 \times 10^{-18}$. In contrast to $K_{\mathrm{c}}$ and $K_{\mathrm{w}}$, the excess equilibrium constant $K_{\mathrm{xs}}$ is independent of the choice of concentration units. We note another important quality of $K_{\mathrm{xs}}$. The reaction equation of water autoprotolysis is often written in the form $2 \mathrm{H}_{2} \mathrm{O} \rightleftharpoons \mathrm{H}_{3} \mathrm{O}^{+}+\mathrm{OH}^{-}$with a hydronium ion in the dissociated state rather than $\mathrm{H}^{+}$. Whereas this yields an entirely different concentration-based equilibrium constant $K_{\mathrm{c}}^{\prime}=\left(c_{\mathrm{H}_{3} \mathrm{O}^{+}} c_{\mathrm{OH}^{-}}\right) /\left(c_{\mathrm{H}_{2} \mathrm{O}, \ell}\right)^{2}=3.3 \times 10^{-18}$ both in magnitude and units, the excess equilibrium constant remains mostly unaffected, $K_{\mathrm{xs}}^{\prime}=\left(1 / g_{\mathrm{r}}^{\prime}\right)\left(c_{\mathrm{H}_{3} \mathrm{O}^{+}} c_{\mathrm{OH}^{-}}\right) /\left(c_{\mathrm{H}_{2} \mathrm{O}, \ell}\right)^{2}$. Only the combinatorial factor $g_{\mathrm{r}}^{\prime}=2 / 3$ is slightly changed with an unimportant effect on the value of $K_{\mathrm{xs}}^{\prime}$. The intrinsic physical information contained in the excess equilibrium constant of water autoprotolysis and the corresponding excess Gibbs free energy of reaction $\Delta_{\mathrm{r}} G_{\mathrm{xs}}^{\mathrm{eq}}=-k_{\mathrm{B}} T \ln \left(K_{\mathrm{xs}}\right)=1.052 \mathrm{eV}$ is discussed in detail in Part II. of this series, cf. Figure II.3(b) therein.

\section{Rate Equations and Rate Constant}

We next analyze the kinetics of real association/dissociation reactions from the perspective of the ideal gas reference. The rate of a bimolecular elementary reaction $\mathrm{A}+\mathrm{B} \rightarrow \mathrm{AB}$ would conventionally be written in the form $R=c_{\mathrm{A}} c_{\mathrm{B}} k^{(2)}$ using a second-order rate constant $k^{(2)}$. Comparison with Eqs. (16) and (23) shows that $k^{(2)}=k_{\mathrm{id}} / c_{\mathrm{ch}}$ for the ideal association reaction, and $k^{(2)}=\left(k_{\mathrm{id}} / c_{\mathrm{ch}}\right) e^{-E_{\mathrm{TS}} / k_{\mathrm{B}} T}$ for the isotropic TST model. We thus 
find a renormalization scheme to reduce the second-order rate constant $k^{(2)}$ to an effective first-order rate constant $k_{\mathrm{eff}}^{(1)}=c_{\mathrm{ch}} k^{(2)}$. For the ideal case, $k_{\mathrm{eff}}^{(1)}=k_{\text {id }}$ is precisely equal to the unimolecular rate constant of the reverse reaction, which led us to introduce the ideal rate constant $k_{\text {id }}$ of Eq. (17) to describe both reaction directions at once. We therefore define the excess rate constant of a bimolecular elementary reaction $k_{\mathrm{xs}}=k_{\mathrm{eff}}^{(1)} / k_{\mathrm{id}}=c_{\mathrm{ch}} k^{(2)} / k_{\mathrm{id}}$. Naturally, the excess rate constant of an ideal bimolecular reaction is equal to one. For the isotropic TST model, we find $k_{\text {eff }}^{(1)}=k_{\mathrm{id}} e^{-E_{\mathrm{TS}} / k_{\mathrm{B}} T}$, and $k_{\mathrm{xS}}=e^{-E_{\mathrm{TS}} / k_{\mathrm{B}} T}$ is simply equal to the Boltzmann factor of the potential energy barrier.

As discussed before, the probability of an elementary reaction with molecularity $\geq 3$ is zero in ideal gas mixtures. However, although rare, non-ideal termolecular reactions exist. For a model of an elementary reaction $\mathrm{A}+\mathrm{B}+\mathrm{C} \rightarrow \mathrm{ABC}$ with short-range attractive pairwise potentials, $\mathrm{Smith}^{4}$ derived a three-body collision rate $R_{\text {coll }}^{(3)}=c_{\mathrm{A}} c_{\mathrm{B}} c_{\mathrm{C}} k_{\text {coll }}^{(3)}$ with an approximate rate constant $k_{\text {coll }}^{(3)}=\pi^{3} \ell^{5} \sqrt{k_{\mathrm{B}} T / 2 \pi \mu^{(3)}}$, where $\ell$ is the collision radius and $\mu^{(3)}=\sqrt{m_{\mathrm{A}} m_{\mathrm{B}} m_{\mathrm{C}} /\left(m_{\mathrm{A}}+m_{\mathrm{B}}+m_{\mathrm{C}}\right)}$ is the reduced mass of the three-body system. For $\ell$ of the order of few $\AA$, we obtain a room-temperature estimate $k_{\text {coll }}^{(3)} \approx 10^{-33} \mathrm{~s}^{-1} \mathrm{~cm}^{6}$, which is entirely different both in magnitude and units from the two-body collision rate constant $k_{\text {coll }}^{(2)} \approx$ $10^{-10} \mathrm{~s}^{-1} \mathrm{~cm}^{3}$ estimated further above. We generalize our renormalization apporach to thirdorder rate constants $k^{(3)}$ to define an effective first-order rate constant $k_{\text {eff }}^{(1)}=c_{\mathrm{ch}}^{2} k^{(3)}$ and a corresponding excess rate constant $k_{\mathrm{xs}}=k_{\mathrm{eff}}^{(1)} / k_{\mathrm{id}}=c_{\mathrm{ch}}^{2} k^{(3)} / k_{\mathrm{id}}$. Applied to Smith's threebody collision rate constant, it yields $k_{\text {eff }}^{(1)}=c_{\text {ch }}^{2} k_{\text {coll }}^{(3)}=(3 \pi / 16)\left(3 v_{\mathrm{th}} / 4 \ell_{\mathrm{ch}}\right)=(3 \pi / 16) k_{\mathrm{id}}$, where we identified $\ell=\ell_{\mathrm{ch}}$ and used $c_{\mathrm{ch}}=1 / V_{\mathrm{ch}}=3 /\left(4 \pi \ell_{\mathrm{ch}}^{3}\right)$ and $v_{\mathrm{th}}=\sqrt{8 k_{\mathrm{B}} T / \pi \mu^{(3)}}$. Remarkably, we find that the renormalized three-body collision rate constant is again approximately equal to the ideal rate constant, and the corresponding excess rate constant $k_{\mathrm{xs}}=k_{\mathrm{eff}}^{(1)} / k_{\mathrm{id}}=3 \pi / 16$ is of order unity. The ideal gas referencing scheme thus produces consistently comparable renormalized rate constants from otherwise incompatible conventional rate constants for reactions with different molecularities.

The unimolecular rate equations, Eqs. (15) and (26), reveal another important aspect. Conventionally, the rate of a unimolecular decay reaction $\mathrm{AB} \rightarrow \mathrm{A}+\mathrm{B}$ would be written $R=c_{\mathrm{AB}} k^{(1)}$ with a first-order rate constant $k^{(1)}$. Comparison with Eqs. (15) and (26) shows that $k^{(1)}=\left(c_{*} / c_{\mathrm{ch}}\right) k_{\mathrm{id}}$ for the ideal association reaction and $k^{(1)}=\left(c_{*} / c_{\mathrm{ch}}\right) k_{\mathrm{id}} e^{-\frac{E_{\mathrm{TS}}-E_{\mathrm{AB}}}{k_{\mathrm{B}} T}}$ for the isotropic TST model. In both cases, we find an additional factor $c_{*} / c_{\mathrm{ch}}=e^{-\left(c_{\mathrm{tot}} / c_{\mathrm{ch}}\right)}$, where $c_{*}$ is the concentration of unoccupied chemical volume elements, making Eqs. (15) and 
(26) effectively appear to be of second order. In the dilute limit, $\left(c_{*} / c_{\mathrm{ch}}\right)$ turns to one and can be neglected. However, in general this factor has a profound influence on the structure and symmetry of the reaction and rate equations. We remind the reader that $c_{*} / c_{\mathrm{ch}}=e^{-\left(c_{\mathrm{tot}} / c_{\mathrm{ch}}\right)}$ is the probability of finding an empty chemical volume element. Two such empty volume elements are required to accommodate both of the free product atoms, whereas only one chemical volume element gets available from the decaying molecule. Therefore, a decay event $\mathrm{AB} \rightarrow \mathrm{A}+\mathrm{B}$ requires one additional chemical volume element, which produces the factor $c_{*} / c_{\mathrm{ch}}$. Effectively, the reaction thus reads $\mathrm{AB}+* \rightarrow \mathrm{A}+\mathrm{B}$, where $*$ denotes an empty chemical volume element $V_{\mathrm{ch}}$. In this form of the reaction equation, the number of reactants and products is balanced by an appropriate number of $*$. This equation structure is the same as encountered for surface reactions that proceed at a limited number of surface sites, as further elaborated on below.

We find that the ideal gas reference for association/dissociation reactions can be described as chemistry on a lattice. The lattice is defined by the chemical volume elements $V_{\mathrm{ch}}$ that produce a chemical partitioning of space. Each molecular entity occupies one lattice site $V_{\mathrm{ch}}$. However, these lattice sites move together with the molecular entities, and the chemical lattice defined by the ideal gas reference is therefore disordered and dynamical. Furthermore, the number of sites $N_{\mathrm{ch}}$ is very large, so that the reservoir of unoccupied sites $*$ is typically large, especially in dilute gas mixtures. In such cases, the corresponding concentration $c_{*}$ is close to the total concentration of chemical volume elements $c_{\mathrm{ch}}$, and the factors $c_{*} / c_{\mathrm{ch}}$ can be neglected.

We now generalize our insight into the structure of the rate equations for the unimolecular, bimolecular, and termolecular cases to a general elementary reaction $\sum_{i} \rho_{i} \mathrm{R}_{i} \rightleftarrows \sum_{j} \pi_{j} \mathrm{P}_{j}$ with molecularities $n=\sum_{i} \rho_{i}$ and $m=\sum_{j} \pi_{j}$ in the forward and backward direction, respectively. If $m>n$, the number of molecular entities produced in the forward direction is larger than the number of molecular entities consumed. As discussed for unimolecular dissociation, we need to include a factor $c_{*} / c_{\mathrm{ch}}$ for each of the additional molecular entities created, so $\left(c_{*} / c_{\mathrm{ch}}\right)^{m-n}$ for the forward rate, and none for the backward rate. Vice versa, if $m<n$, we include a factor $\left(c_{*} / c_{\mathrm{ch}}\right)^{n-m}$ for the backward rate, and none for the forward rate. Effectively, we can write the general reaction equation in the form $\sum_{i} \rho_{i} \mathrm{R}_{i}+\rho_{*} * \rightleftarrows$ $\sum_{j} \pi_{j} \mathrm{P}_{j}+\pi_{*} *$, where $\rho_{*}=\max (m-n, 0)$ and $\pi_{*}=\max (n-m, 0)$ with $\max (\ldots)$ denoting 
the greater of the two arguments. Accordingly, we write the forward and backward rates as

$$
R_{\mathrm{f}}=k_{\mathrm{xs}}^{\mathrm{f}} k_{\mathrm{id}} c_{\mathrm{ch}}\left(\frac{c_{*}}{c_{\mathrm{ch}}}\right)^{\rho_{*}} \prod_{i}\left(\frac{c_{\mathrm{R}_{i}}}{c_{\mathrm{ch}}}\right)^{\rho_{i}}
$$

and

$$
R_{\mathrm{b}}=k_{\mathrm{xs}}^{\mathrm{b}} k_{\mathrm{id}} c_{\mathrm{ch}}\left(\frac{c_{*}}{c_{\mathrm{ch}}}\right)^{\pi_{*}} \prod_{j}\left(\frac{c_{\mathrm{P}_{j}}}{c_{\mathrm{ch}}}\right)^{\pi_{j}}
$$

respectively, with corresponding excess rate constants $k_{\mathrm{xs}}^{\mathrm{f}}$ and $k_{\mathrm{xs}}^{\mathrm{b}}$. Note that the forward and backward rate equations are entirely symmetric with an identical number of $\max (n, m)$ concentration factors. Conventionally, these rate equations would be written in the form $R_{\mathrm{f}}=k_{\mathrm{f}}^{(n)} \prod_{i}\left(c_{\mathrm{R}_{i}}\right)^{\rho_{i}}$ and $R_{\mathrm{b}}=k_{\mathrm{b}}^{(m)} \prod_{j}\left(c_{\mathrm{P}_{j}}\right)^{\pi_{j}}$ with an $n$ th-order rate constant $k_{\mathrm{f}}^{(n)}$ and an $m$ th-order rate constant $k_{\mathrm{b}}^{(m)}$. Comparison with Eqs. (40) and (41) yields the general relation between the dimensionless excess rate constant $k_{\mathrm{xs}}$ and the conventional $n$ th-order rate constant $k^{(n)}$,

$$
k_{\mathrm{xs}}=\left(\frac{c_{\mathrm{ch}}^{n-1} k^{(n)}}{k_{\mathrm{id}}}\right)\left(\frac{c_{\mathrm{ch}}}{c_{*}}\right)^{\max (m-n, 0)},
$$

where the first factor $\left(c_{\mathrm{ch}}^{n-1} k^{(n)} / k_{\mathrm{id}}\right)$ is dominant. At equilibrium, $R_{\mathrm{f}}=R_{\mathrm{b}}$, we find from Eqs. (40) and (41)

$$
\frac{k_{\mathrm{xs}}^{\mathrm{f}}}{k_{\mathrm{xs}}^{\mathrm{b}}}=\frac{\prod_{j}\left(c_{\mathrm{P}_{j}}^{\mathrm{eq}}\right)^{\pi_{j}}}{\prod_{i}\left(c_{\mathrm{R}_{i}}^{\mathrm{eq}}\right)^{\rho_{i}}} \frac{\left(c_{*}\right)^{\sum_{i} \rho_{i}}}{\left(c_{*}\right)^{\sum_{j} \pi_{j}}}=g_{\mathrm{r}} K_{\mathrm{xs}} .
$$

The ratio of the excess rate constants of forward over backward reaction is equal to the excess equilibrium constant $K_{\mathrm{xs}}$ of the reaction, cf. Eq. (35), multiplied by the combinatorial factor $g_{\mathrm{r}}$ that is typically of order unity. This demonstrates the consistency of the ideal gas reference in terms of both equilibrium and kinetics.

Based on our discussion of the unimolecular, bimolecular, and termolecular cases, we consider $k_{\mathrm{xs}}$ as a measure of the "ideality" of the kinetics. In cases where the reaction rate is limited by an activation energy barrier, $k_{\mathrm{xs}}$ is essentially determined by the corresponding Boltzmann factor, yielding $k_{\mathrm{xs}}<1$. For $k_{\mathrm{xs}} \rightarrow 1$ approaching ideal kinetics, the rate of reaction becomes limited by the thermal velocity of the reactants. Ideal kinetics thus represent an upper bound for the velocity of most real association/dissociation reactions. Exceptions from that rule, however, could exist in cases where long-range attractive forces act between the reactants, in combination with a negligible activation energy barrier. The 
effective collision cross section would then be larger than the molecular cross section, resulting in super-ideal kinetics with $k_{\mathrm{xs}}>1$. Nevertheless, we regard ideal kinetics as an effective upper bound for the reaction velocity in most practical cases. Taking into account $c_{*}<c_{\mathrm{ch}}$ in Eq. (42) and setting $k_{\mathrm{xs}} \leq 1$, we obtain an upper bound estimate for the $n$ th-order rate constant $k^{(n)} \leq\left(k_{\mathrm{id}} / c_{\mathrm{ch}}^{n-1}\right)$. If we further consider $c_{\mathrm{ch}}$ as an upper bound for any of the reactant concentrations, we find an overall upper bound for the rate of an elementary reaction $R \leq c_{\mathrm{ch}}^{n}\left(k_{\mathrm{id}} / c_{\mathrm{ch}}^{n-1}\right)=c_{\mathrm{ch}} k_{\mathrm{id}}=R_{\max }$ which is the same for any molecularity $n$. For $\ell_{\mathrm{ch}}$ of the order of few $\AA$, we have $c_{\mathrm{ch}} \approx 10-100 \mathrm{~mol} \mathrm{~L}^{-1}$. Together with our room-temperature estimate $k_{\mathrm{id}} \approx 10^{12}-10^{13} \mathrm{~s}^{-1}$, we obtain $R_{\max } \approx 10^{13}-10^{15} \mathrm{~mol} \mathrm{~L}^{-1} \mathrm{~s}^{-1}$.

So far, we restricted the discussion to the kinetics of elementary reactions. For stepwise reactions, no general form of an overall rate law exists due to the nonlinearity of the system of rate equations of all elementary steps. Nevertheless, excess rate constants can be defined for each of the individual steps. Furthermore, many multistep reactions still yield an overall rate law of the simple form (40) with integer reaction orders. This is the case, e.g., when either the first step is rate determining, or when there are no "spectator" intermediates during the rate-determining step (rds) that do not participate in the rds. In such cases, an overall excess rate constant $k_{\mathrm{xs}}^{\mathrm{tot}}=K_{\mathrm{xs}}^{(0, \mathrm{rds})} k_{\mathrm{xs}}^{\mathrm{rds}}$ of the multistep reaction can be defined that is given by the product of the excess rate constant $k_{\mathrm{xs}}^{\mathrm{rds}}$ of the rds and the excess equilibrium constant $K_{\mathrm{xs}}^{(0, \mathrm{rds})}$ of all reaction steps preceding the rds.

Whereas conventional rate constants of reactions with differing reaction orders cannot be compared, excess rate constants enable comparison of kinetics across different reaction orders. As an example, we again consider the water autoprotolysis reaction $\mathrm{H}_{2} \mathrm{O} \rightleftharpoons \mathrm{H}^{+}+$ $\mathrm{OH}^{-}$. The second-order rate constant of the hydronium-hydroxide recombination reaction was reported ${ }^{5}$ with a value $k_{\text {rec }}^{(2)}=1.3 \times 10^{11} \mathrm{~s}^{-1}\left(\mathrm{~mol} \mathrm{~L}^{-1}\right)^{-1}$. The corresponding excess rate constant is $k_{\mathrm{xs}}^{\mathrm{rec}}=c_{\mathrm{ch}} k_{\mathrm{rec}}^{(2)} / k_{\mathrm{id}}$ according to Eq. (42). The aqueous value of $\ell_{\mathrm{ch}} \approx 1.5-1.6 \AA$, discussed above, corresponds to $c_{\mathrm{ch}} \approx 100 \mathrm{~mol} \mathrm{~L}{ }^{-1}$, which yields $k_{\mathrm{xs}}^{\mathrm{rec}} \approx\left(1.3 \times 10^{13} \mathrm{~s}^{-1}\right) / k_{\mathrm{id}} \approx$ 1 for a value of $k_{\mathrm{id}} \approx 10^{13} \mathrm{~s}^{-1}$ at room temperature as estimated before. We find that the hydronium-hydroxide recombination kinetics are essentially ideal, characterized by an excess rate constant close to unity. The first-order rate constant $k_{\text {diss }}^{(1)}$ of the water dissociation direction is obtained from the requirement that the total water dissociation rate $R_{\text {diss }}=$ $c_{\mathrm{H}_{2} \mathrm{O}, \ell} k_{\text {diss }}^{(1)}$ must be equal to the total recombination rate $R_{\mathrm{rec}}=c_{\mathrm{H}^{+}} c_{\mathrm{OH}^{-}} k_{\mathrm{rec}}^{(2)}=1.3 \times$ $10^{-3} \mathrm{~mol} \mathrm{~L}^{-1} \mathrm{~s}^{-1}$ at equilibrium. With $c_{\mathrm{H}_{2} \mathrm{O}, \ell}=55.34 \mathrm{~mol} \mathrm{~L}^{-1}$, we obtain $k_{\text {diss }}^{(1)}=2.3 \times 10^{-5} \mathrm{~s}^{-1}$, 
which corresponds to an excess rate constant of $k_{\mathrm{xs}}^{\mathrm{diss}}=\left(k_{\mathrm{diss}}^{(1)} / k_{\mathrm{id}}\right)\left(c_{\mathrm{ch}} / c_{*}\right)$ according to Eq. (42) with $m=2$ and $n=1$. With $c_{*}=c_{\mathrm{H}_{2} \mathrm{O}, \ell}=55.34 \mathrm{~mol} \mathrm{~L}^{-1}$, as before, we find $k_{\mathrm{xs}}^{\text {diss }} \approx 4 \times 10^{-18}$. Whereas the recombination kinetics were found to be essentially ideal, the dissociation kinetics are extremely slowed down by the difference in potential energy between the dissociated product state and the molecular water reactant. This aspect is further discussed in Part II. of this series, cf. Figure II.3(b) therein.

\section{B. Surface Reactions}

The rate and equilibrium equations obtained from the ideal gas framework naturally include the concentration $c_{*}$ of unoccupied chemical volume elements. Furthermore, all concentration factors $c_{i} / c_{\mathrm{ch}}$ in the general rate equation (40) are referenced to the total concentration $c_{\mathrm{ch}}$ of chemical volume elements. These factors can be interpreted as coverages $\omega_{i}=c_{i} / c_{\mathrm{ch}}=N_{i} / N_{\mathrm{ch}}$ on a "chemical lattice" with a total number of $N_{\mathrm{ch}}$ sites, each of which representing one chemical volume element $V_{\mathrm{ch}}$. In particular, the rate equation (40) of a general volume reaction can be written in the form

$$
R=R_{\max } k_{\mathrm{xs}}\left(\omega_{*}\right)^{\rho_{*}} \prod_{i}\left(\omega_{\mathrm{R}_{i}}\right)^{\rho_{i}}
$$

where $R_{\max }=c_{\mathrm{ch}} k_{\mathrm{id}}=\left(N_{\mathrm{ch}} / V\right) k_{\mathrm{id}}$ is the upper bound for the volume-specific reaction rate.

We find that the ideal gas reference for general association/dissociation reactions yields equations of the same structure as those of surface reactions proceeding on a lattice of surface sites. Whereas the reactions considered so far are described by volume-specific rates, i.e. number of reactions per time and volume, surface reactions are described by area-specific rates. Assuming a surface with area $\mathcal{A}$ subdivided into a total number $N_{\mathrm{s}}$ of sites, we generalize the ideal gas reference to a surface reaction

$$
\sum_{p} \sigma_{p} \mathrm{~S}_{p}+\sigma_{*} *_{\mathrm{s}}+\sum_{i} \rho_{i} \mathrm{R}_{i}+\rho_{*} *_{\mathrm{ch}} \rightleftarrows \sum_{q} \tau_{q} \mathrm{~T}_{q}+\tau_{*} *_{\mathrm{s}}+\sum_{j} \pi_{j} \mathrm{P}_{j}+\pi_{*} *_{\mathrm{ch}}
$$

where $\mathrm{S}_{p}$ and $\mathrm{T}_{q}$ denote reactant and product species, respectively, that are adsorbed to, i.e. occupying, surface sites, and $\sigma_{p}$ and $\tau_{q}$ are the corresponding stoichiometric coefficients. The coefficients $\sigma_{*}$ and $\tau_{*}$ account for the net number of unoccupied surface sites $\left(*_{\mathrm{s}}\right)$ becoming either occupied $\left(\sigma_{*}>0\right)$ or liberated $\left(\tau_{*}>0\right)$ during the reaction. The non-adsorbed gasphase species $\mathrm{R}_{i}, \mathrm{P}_{j}$, coefficients $\rho_{i}, \rho_{*}, \pi_{j}, \pi_{*}$, and unoccupied chemical volume elements 
$*_{\text {ch }}$ are defined as before in the context of Eqs. (40) and (41). The area-specific rates of the forward and backward reaction can be written in the form

$$
r_{\mathrm{f}}=r_{\max } k_{\mathrm{xs}}^{\mathrm{f}}\left(\theta_{*_{\mathrm{s}}}\right)^{\sigma_{*}}\left(\omega_{*_{\mathrm{ch}}}\right)^{\rho_{*}} \prod_{p}\left(\theta_{\mathrm{S}_{p}}\right)^{\sigma_{p}} \prod_{i}\left(\omega_{\mathrm{R}_{i}}\right)^{\rho_{i}}
$$

and

$$
r_{\mathrm{b}}=r_{\max } k_{\mathrm{xs}}^{\mathrm{b}}\left(\theta_{*_{\mathrm{s}}}\right)^{\tau_{*}}\left(\omega_{*_{\mathrm{ch}}}\right)^{\pi_{*}} \prod_{q}\left(\theta_{\mathrm{T}_{q}}\right)^{\tau_{q}} \prod_{j}\left(\omega_{\mathrm{P}_{j}}\right)^{\pi_{j}}
$$

respectively, where $r_{\max }=\left(N_{\mathrm{s}} / \mathcal{A}\right) k_{\mathrm{id}}$ is the upper bound for the area-specific reaction rate, analogous to $R_{\max }$ in Eq. (44). All reactant and product species, both adsorbed and non-adsorbed, enter these rate equations via coverage factors, either in the form of surface coverages $\theta_{i}=N_{i} / N_{\mathrm{s}}$ or volume coverages $\omega_{i}=N_{i} / N_{\mathrm{ch}}$. Likewise, the unoccupied surface sites and the unoccupied chemical volume elements appear in the form of the empty-site fractions $\theta_{*_{\mathrm{s}}}$ and $\omega_{*_{\mathrm{ch}}}$, respectively. Note that the ideal rate constant $k_{\mathrm{id}}$ in $r_{\max }$ is the same as before, being equal to the inverse of the average time that it takes for an ideal gas particle at thermal velocity to traverse the volume element defined by a surface site. Therefore, in the context of surface reactions, $k_{\mathrm{id}}$ can also be interpreted as the ideal turnover frequency (TOF) per surface site. As discussed before, its value is in the range of few $\mathrm{THz}$ at room temperature.

At equilibrium, $r_{\mathrm{f}}=r_{\mathrm{b}}$, we obtain the excess equilibrium constant of the surface reaction, as in Eq. (43),

$$
K_{\mathrm{xS}}=\frac{1}{g_{\mathrm{r}}} \frac{k_{\mathrm{xs}}^{\mathrm{f}}}{k_{\mathrm{xS}}^{\mathrm{b}}}=\left(\frac{1}{g_{\mathrm{r}}}\right) \frac{\left(\theta_{*_{\mathrm{s}}}\right)^{\sum_{p} \sigma_{p}} \prod_{q}\left(\theta_{\mathrm{T}_{q}}^{\mathrm{eq}}\right)^{\tau_{q}}}{\left(\theta_{*_{\mathrm{s}}}\right)^{\sum_{q} \tau_{q}} \prod_{p}\left(\theta_{\mathrm{S}_{p}}\right)^{\mathrm{eq}_{p}}} \frac{\left(\omega_{*_{\mathrm{ch}}}\right)^{\sum_{i} \rho_{i}} \prod_{j}\left(\omega_{\mathrm{P}_{j}}^{\mathrm{eq}}\right)^{\pi_{j}}}{\left(\omega_{*_{\mathrm{ch}}}\right)^{\sum_{j} \pi_{j}} \prod_{i}\left(\omega_{\mathrm{R}_{i}}^{\mathrm{eq}}\right)^{\rho_{i}}}
$$

with the net combinatorial factor $g_{\mathrm{r}}=\left[\prod_{p}\left(g_{\mathrm{S}_{p}}\right)^{\sigma_{p}} \prod_{i}\left(g_{\mathrm{R}_{i}}\right)^{\rho_{i}}\right] /\left[\prod_{q}\left(g_{\mathrm{T}_{q}}\right)^{\tau_{q}} \prod_{j}\left(g_{\mathrm{P}_{j}}\right)^{\pi_{j}}\right]$ of the reaction, where the combinatorial factors $g_{i}$ for each species are defined as before. The number of coverage factors in the numerator and denominator is again balanced by the fractions of unoccupied surface sites and chemical volume elements.

The ideal gas reference leads to a symmetric description of volume and surface reactions, with the chemical volume elements $V_{\text {ch }}$ being the volume equivalent of the surface sites. The main difference between gas phase reactions and surface reactions is the availability of empty sites. For volume reactions in the gas phase, the number of volume sites $N_{\text {ch }}$ is generally 
much larger than the number of molecular entities present. Therefore, the volume coverages $\omega_{i} \ll 1$ are typically small and the fraction of unoccupied chemical volume elements $\omega_{*_{\mathrm{ch}}} \approx 1$ is close to one, with the consequence that the $\omega_{*_{\mathrm{ch}}}$ factors in the respective rate equations can usually be neglected. For surface reactions, in contrast, the number of surface sites $N_{\mathrm{s}}$ is generally limiting and the surface coverages $\theta_{i}$ are anything between zero and one. The fraction of unoccupied surface sites $\theta_{*_{\mathrm{s}}}$ is often significantly smaller than one and can have a strong influence on the reaction rates.

To maximize the rate of an elementary surface reaction step, described by a rate equation (46), all the involved surface coverages must not be too small, and therefore of a similar magnitude. On the contrary, if one adsorbate species has a dominating binding energy, its coverage becomes close to one and all the other coverages are close to zero, making the product of coverages in the rate equation extremely small. This is the well-known Sabatier principle of heterogeneous catalysis, which requires a fine balance between the binding energies of all adsorbed species for an optimal catalyst, resulting in approximately equal surface coverages of all involved species. The requirement of balanced binding energies corresponds to an alignment of the various reaction intermediates at a similar "height" within the potential energy surface. As further discussed below, the ideal gas reference is equivalent to the limit of an entirely flat potential energy landscape with all states being aligned. Therefore, the Sabatier principle is closely related to the ideal gas reference, with the energetic alignment of reaction intermediates being quantified by the respective excess equilibrium constants.

\section{Physical Interpretation}

Excess constants. The ideal gas reference yields rate and equilibrium equations in a consistent form regardless of the molecularity and reaction orders involved. Beyond this aesthetic quality, we also expect a practical benefit by enabling comparison between reactions with otherwise incompatible rate and equilibrium constants. The dimensionless excess constants could thus be useful descriptors, e.g., for machine learning applications in chemistry.

Furthermore, the excess equilibrium and rate constants have a comprehensible physical meaning. From the discussion of the isotropic TST model, we saw that the ideal gas reference 


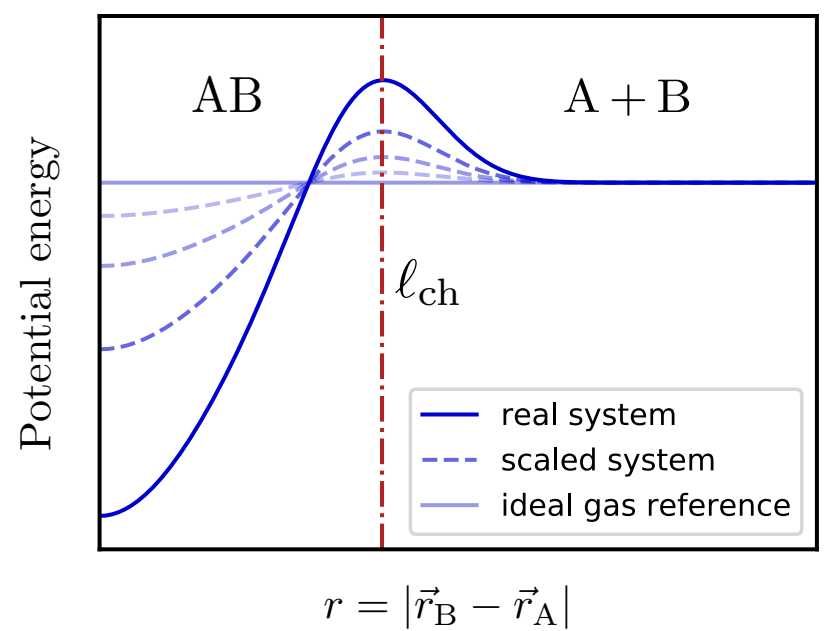

FIG. 2. Schematic of the potential energy of a system of atoms A and B as a function of their distance. The potential energy is negative in the bound molecular state AB that is separated by a positive potential energy barrier from the dissociated state. A natural choice for $\ell_{\mathrm{ch}}$ is the distance between $\mathrm{A}$ and $\mathrm{B}$ at the transition state, indicated by a vertical line. Scaling the potential energy unevenness down to zero (indicated by the dashed curves), whilst preserving $\ell_{\mathrm{ch}}$, yields the ideal gas reference for association/dissociation that corresponds to an entirely flat potential energy landscape (horizontal solid line).

corresponds to the limit of an entirely flat potential energy landscape. In this sense, our initial, somewhat artificial definition of a molecular entity based on an arbitrarily chosen critical distance $\ell_{\mathrm{ch}}$ is justified in hindsight as being simply the zero barrier limit of the intuitive notion of a molecular entity being held together by a certain binding energy and separated by its surroundings through a potential energy barrier, as schematically shown in Figure 2. The excess equilibrium and rate constants of a real reaction, which are defined by normalizing the actual constants with those of the ideal gas reference, quantify the systemspecific effects resulting from the unevenness of the potential energy surface. All aspects that are independent of the shape of the potential energy landscape, and thus unspecific, are characterized by the ideal gas reference.

Choice and meaning of $\ell_{\mathrm{ch}}$. Initially, $\ell_{\mathrm{ch}}$ was introduced as an arbitrarily chosen critical distance defining when non-interacting atomic constituents are considered to belong together and form a molecular entity. We then found that the same formalism of association/dissociation chemistry in ideal gases is obtained from the limit of a real system where 
all unevenness of the potential energy surface is scaled down to zero, as schematically visualized in Figure 2. Performing this limit, it becomes clear that a natural choice for $\ell_{\mathrm{ch}}$ is the distance between atomic constituents at the transition state for association/dissociation, as indicated by the vertical line in Figure 2. Since this depends on the molecule at hand, it appears appropriate to define different $\ell_{\mathrm{ch}}$ for different molecules to account for differences in molecular size. The molecular volume $V_{\mathrm{ch}}$ and the reference concentration $c_{\mathrm{ch}}=1 / V_{\mathrm{ch}}$ would then become species-dependent in the general rate equations (40) and (41). Such a distinction could, of course, be included in the definition of the excess equilibrium and rate constants. However, variations in molecular volumes and bond lengths are typically of order unity, and therefore unimportant in an analysis of equilibrium and rate constants that typically vary by orders of magnitude. We therefore consider it most practical to choose a common value for $\ell_{\mathrm{ch}}$ at a certain average radius of the species involved in a given reaction.

We finally note that $\ell_{\mathrm{ch}}$ introduces a chemical length scale. If the average distance between molecules is large compared to $\ell_{\mathrm{ch}}$, each molecule can be well distinguished from the others, representing the dilute gas limit. Conversely, if the average distance between molecules is close to $\ell_{\mathrm{ch}}$, which happens when the concentrations turn towards $c_{\mathrm{ch}}$, the distinction of molecular entities becomes ambiguous and the system reaches the condensed matter limit. It is interesting to note the analogy between the chemical length scale $\ell_{\mathrm{ch}}$ and the thermal wavelength $\lambda_{\text {th }}$ that defines a critical concentration $c_{\text {th }}=1 / \lambda_{\text {th }}^{3}$ above which the classical picture of distinct individual particles fails and the quantum mechanical wave character becomes manifest.

\section{CONCLUSIONS}

The definition of molecular entities in mixtures of ideal gases provides an ideal gas reference for chemical association and dissociation reactions. This framework leads to an ideal law of mass action and ideal kinetic rate equations, characterized by ideal equilibrium and rate constants, respectively. These are the basis for the definition of excess equilibrium and rate constants for real reactions, which quantify the deviation from ideal behavior. Unlike the conventional equilibrium and rate constants, their excess counterparts enable consistent comparison across reactions with different reaction orders. Moreover, the ideal gas reference is precisely obtained when scaling all unevenness in the potential energy surface of a real 
system down to zero. The excess constants thus quantify the effect of the system-specific shape of the potential energy landscape on equilibria and kinetics of association/dissociation reactions.

\section{SUPPLEMENTARY MATERIAL}

See supplementary material for the calculation of relative thermal velocities.

\section{ACKNOWLEDGMENTS}

T.B. acknowledges financial support in the form of a research fellowship grant funded by the SNSF (Swiss National Science Foundation). R.M. acknowledges financial support from the South African Department of Science and Innovation in the form of HySA/Catalysis Centre of Competence programme funding.

\section{AUTHOR DECLARATIONS}

The authors have no conflicts to disclose.

\section{DATA AVAILABILITY STATEMENT}

Data sharing is not applicable to this article as no new data were created or analyzed in this study.

\section{REFERENCES}

${ }^{1}$ A. D. McNaught and A. Wilkinson, eds., IUPAC. Compendium of Chemical Terminology, 2nd ed. (the "Gold Book") (Blackwell Scientific Publications, Oxford, 1997) online version (2019-) created by S. J. Chalk.

${ }^{2}$ H. Eyring, "The activated complex in chemical reactions," The Journal of Chemical Physics 3, 107-115 (1935). 
${ }^{3}$ P. J. Linstrom and W. G. Mallard, eds., NIST Chemistry WebBook, NIST Standard Reference Database Number 69 (National Institute of Standards and Technology, Gaithersburg MD, 20899, 2018) retrieved May 17, 2020.

${ }^{4}$ F. T. Smith, "Three-body collision rates in atomic recombination reactions," Discuss. Faraday Soc. 33, 183-188 (1962).

${ }^{5}$ M. Eigen and L. De Maeyer, "Untersuchungen über die kinetik der neutralisation. i," Zeitschrift für Elektrochemie, Berichte der Bunsengesellschaft für physikalische Chemie 59, 986-993 (1955). 